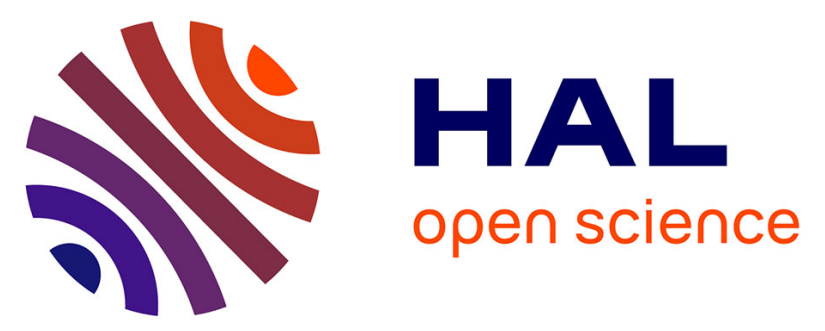

\title{
Protease Inhibitors. Part 2. Weakly Basic Thrombin Inhibitors Incorporating Sulfonyl-Aminoguanidine Moieties as S1 Anchoring Groups: Synthesis and Structure-Activity Correlations
}

Brian Clare, Andrea Scozzafava, Fabrizio Briganti, Bogdan Iorga, Claudiu Supuran

\section{To cite this version:}

Brian Clare, Andrea Scozzafava, Fabrizio Briganti, Bogdan Iorga, Claudiu Supuran. Protease Inhibitors. Part 2. Weakly Basic Thrombin Inhibitors Incorporating Sulfonyl-Aminoguanidine Moieties as S1 Anchoring Groups: Synthesis and Structure-Activity Correlations. Journal of Enzyme Inhibition and Medicinal Chemistry, 2000, 15 (3), pp.235-264. 10.3109/14756360009040686 . hal-03161392

\section{HAL Id: hal-03161392 \\ https://hal.science/hal-03161392}

Submitted on 6 Mar 2021

HAL is a multi-disciplinary open access archive for the deposit and dissemination of scientific research documents, whether they are published or not. The documents may come from teaching and research institutions in France or abroad, or from public or private research centers.
L'archive ouverte pluridisciplinaire HAL, est destinée au dépôt et à la diffusion de documents scientifiques de niveau recherche, publiés ou non, émanant des établissements d'enseignement et de recherche français ou étrangers, des laboratoires publics ou privés. 


\title{
Protease Inhibitors. Part 2. Weakly Basic Thrombin Inhibitors Incorporating Sulfonyl-Aminoguanidine Moieties as S1 Anchoring Groups: Synthesis and Structure-Activity Correlations
}

\author{
Brian W. Clare, Andrea Scozzafava, Fabrizio Briganti, Bogdan Iorga \& Claudiu \\ T. Supuran
}

To cite this article: Brian W. Clare, Andrea Scozzafava, Fabrizio Briganti, Bogdan lorga \& Claudiu T. Supuran (2000) Protease Inhibitors. Part 2. Weakly Basic Thrombin Inhibitors Incorporating Sulfonyl-Aminoguanidine Moieties as S1 Anchoring Groups: Synthesis and Structure-Activity Correlations, Journal of Enzyme Inhibition, 15:3, 235-264, DOI: 10.3109/14756360009040686

To link to this article: http://dx.doi.org/10.3109/14756360009040686

曲 Published online: 27 Sep 2008.

Submit your article to this journal $₫$

凹 Article views: 12

Q View related articles $\sqsubset$

Citing articles: 1 View citing articles ¿ð 


\title{
PROTEASE INHIBITORS. PART 2. WEAKLY BASIC THROMBIN INHIBITORS INCORPORATING SULFONYL- AMINOGUANIDINE MOIETIES AS S1 ANCHORING GROUPS: SYNTHESIS AND STRUCTURE-ACTIVITY CORRELATIONS
}

\author{
BRIAN W. CLARE ${ }^{a, *}$, ANDREA SCOZZAFAVA $^{\mathrm{b}}$, \\ FABRIZIO BRIGANTI ${ }^{b}$, BOGDAN IORGA $^{c}$ \\ and CLAUDIU T. SUPURAN ${ }^{\mathrm{b}}$ ** \\ ${ }^{a}$ Division of Science, Murdoch University, Murdoch, Perth, W.A. 6150, Australia; \\ ${ }^{\mathrm{b}}$ Laboratorio di Chimica Inorganica e Bioinorganica, Università degli Studi, \\ Via Gino Capponi 7, I-50121, Florence, Italy; ' Department of Chemistry, \\ University of Bucharest, Bd. Republicii 13, 70346-Bucharest, Roumania
}

(Received 30 June 1999)

Two series of derivatives have been prepared and assayed as inhibitors of two physiologically relevant serine proteases, human thrombin and human trypsin. The first series includes alkyl- aralkyl-/aryl- and hetarylsulfonyl-aminoguanidines. It was thus observed that sulfanilyl-aminoguanidine possesses moderate but intrinsically selective thrombin inhibitory properties, with $K_{\mathbf{I}}$ values around 90 and $1400 \mathrm{nM}$ against thrombin and trypsin respectively. Further elaboration of this molecule afforded compounds that inhibited thrombin with $K_{\mathrm{I}}$ values in the range $10-50 \mathrm{nM}$, whereas affinity for trypsin remained relatively low. Such compounds were obtained either by attaching benzyloxycarbonyl- or 4-toluenesulfonylureido-protected amino acids (such as $D$-Phe, $L$-Pro) or dipeptides (such as Phe-Pro, Gly-His, $\beta$-Ala-His or Pro-Gly) to the N-4 atom of the lead molecule, sulfanilyl-aminoguanidine, or by attaching substituted-pyridiniumpropylcarboxamido moieties to this lead. Thus, this study brings novel insights regarding a novel non-basic $\mathrm{S} 1$ anchoring moiety (i.e., $\left.\mathrm{SO}_{2} \mathrm{NHNHC}(=\mathrm{NH}) \mathrm{NH}_{2}\right)$, and new types of peptidomimetic scaffolds obtained by incorporating tosylureido-amino acids/pyridinium-substituted-GABA moieties in the hydrophobic binding site(s). Structure-activity correlations of the new serine protease inhibitors are also discussed based on a QSAR model described previously for a large series of structurally-related derivatives (Supuran et al. (1999) J. Med. Chem., in press).

Keywords: Thrombin; Inhibition; Trypsin; Sulfonyl-aminoguanidines

* Corresponding authors. Tel.: +61-8-93602157. Fax: $+61-8-3101711$.

E-mail: clare@chem.murdoch.edu.au. Fax:+39-055-2757555. E-mail: cts@bio.chim.unifi.it. 


\section{INTRODUCTION}

In a previous paper ${ }^{1 \text { a }}$ we showed that arylsulfonyl-guanidines and arylsulfonyl-isoureas possess a relatively weak but specific affinity for the serine protease thrombin, without inhibiting appreciably the other important enzyme of this family, trypsin. Furthermore, a QSAR study of such derivatives explained their biological activity in terms of the global properties of these molecules, the electronic properties of the anchoring moieties to the enzyme, as well as some novel types descriptors (such as the frontier orbital phase angle, FOPA) recently introduced in medicinal chemistry calculations by one of the authors of this paper. ${ }^{1 b}$

In the series of previously mentioned compounds, ${ }^{1 a}$ which possess novel non-basic anchoring moieties to the enzyme (of the type $\mathrm{SO}_{2} \mathrm{~N}=\mathrm{C}\left(\mathrm{NH}_{2}\right)_{2}$ and $\mathrm{SO}_{2} \mathrm{~N}=\mathrm{C}(\mathrm{OMe}) \mathrm{NH}_{2}$ ), it was observed that sulfaguanidine (as well as the corresponding $O$-methylurea derivative) are good lead molecules for further elaboration. Indeed, this widely used sulfadrug and the related sulfanilyl- $O$-methyl-isourea behaved as weak, but selective thrombin inhibitors; with $K_{\mathrm{I}}$ values of around 100 and $1350-1400 \mathrm{nM}$ against thrombin and trypsin respectively.' It appeared thus of great interest to study structurallyrelated systems, incorporating aminoguanidine instead of guanidine/ $O$-methylisourea, and to elaborate eventually this compound (i.e., sulfanilylaminoguanidine) as a lead for obtaining more powerful/selective thrombin inhibitors.

Thrombin (EC 3.4.21.5) has become an important target for drug design in recent years in the search for low molecular weight, potent and selective inhibitors with applications as diagnostic and therapeutic agents for the increasingly common thrombotic diseases. ${ }^{2-6}$ Although a large number of potent active site-directed thrombin inhibitors, such as peptide aldehydes, ${ }^{7,8}$ boronates, ${ }^{9}$ benzamidine- 2.10 .11 or arginine-guanidine-derived ${ }^{12}$ inhibitors have been reported, none of them meets all the criteria needed for an ideal antithrombotic drug. ${ }^{2.13}$ Thus, the largest majority of the presently available low molecular weight inhibitors, such as argatroban (MQPA) $1,{ }^{14}$ inogatran $2{ }^{6}$ NAPAP 3, ${ }^{15}$ 4-TAPAP 4 or its 3-amidino isomer, 3-TAPAP 5, ${ }^{2.15}$ are poorly bioavailable, either due to their high basicity, connected with the presence of guanidino/amidino moieties in their molecule, or are not absorbable orally, or are rapidly eliminated from the circulation, mainly due to their peptidic nature. Although recently some non-basic S1 anchoring groups have been incorporated in the molecules of some thrombin inhibitors, ${ }^{3.5 .16}$ the presence of guanidino benzamidino moieties in such compounds is critical, since it is by means of the interaction of these highly polar groups 
with Asp 189, the central amino acid residue from the specificity pocket, that the enzyme-inhibitor adduct is initially formed (obviously, a lot of other secondary interactions are responsible for the formation of high affinity adducts between thrombin and its inhibitors).$^{3-5,10-12}$ In order to exploit the intrinsically high affinity of guanidino-/benzamidino-containing inhibitors for the thrombin active site, but also to avoid undesired properties connected with their too high basicity, we propose here a novel approach for designing tight-binding such inhibitors by using sulfonylaminoguanidino moieties as anchoring groups to the specificity S1 pocket. Obviously, the presence of the $\mathrm{SO}_{2}$ group in the neighborhood of the aminoguanidino moiety strongly reduces the basicity of the latter, presumably without precluding the binding of inhibitors within the enzyme active site.<smiles>CC1CNc2c(cccc2S(=O)(=O)NC(CCCN[C@H](N)[NH3+])CN2CCC(C)CC2C(=O)[O-])C1</smiles>

1<smiles>N[C+](N)c1ccc(CC(NC(=O)CNS(=O)(=O)c2ccc3ccccc3c2)C(=O)N2CCCCC2)cc1</smiles>

3<smiles>N=C(N)NCCCNC(=O)[C@@H]1CCCCN1C(=O)C(CC1CCCCC1)NCC(=O)O</smiles>

2<smiles>Cc1ccc(S(=O)(=O)NC(Cc2ccc(C(N)=[NH2+])cc2)C(=O)N2CCCCC2)cc1</smiles>

4

In this paper we report the preparation and serine protease inhibitory properties (against human thrombin and human trypsin) of some alkyl-/ aralkyl- or arylsulfonyl-aminoguanidines, obtained by reaction of aminoguanidine with sulfonyl halides. The most promising compound formed 
(from the point of view of its thrombin inhibitory properties, as well as that of its specificity for thrombin over trypsin), i.e., sulfanilyl-aminoguanidine, was then further derivatized, using two different approaches. The first one included the attachment of amino acyl/dipeptidyl moieties to the aminophenyl moiety of this molecule, leading thus to higher affinity thrombin inhibitors with potencies of the same order of magnitude as those of the clinically used compounds argatroban (MQPA) $1,{ }^{14}$ and inogatran $2 .^{6}$ The second approach used by us consisted in attaching substituted-pyridiniumpropylcarboxamido moieties (obtained by reaction of pyrylium salts with GABA) to the same amino group mentioned above, which produced again in some cases compounds with high affinity for thrombin. Structure-activity correlations for the newly synthesized serine protease inhibitors are also discussed. Compounds, both from this group as well as two other similar series, ${ }^{\text {a }}$ have been included in the discussion based only on calculated descriptors, which were indicative for differences in the requirements for trypsin/thrombin inhibition (which might be further exploited for obtaining a higher specificity). The descriptors used included empirical quantities such as lipophilicity, and quantum chemical indices such as atomic Mulliken charges and superdelocalizabilities, and global quantum indices such as orbital energies, dipole moments, static field polarizabilities, and the new frontier orbital phase angles. ${ }^{\text {tb }}$

\section{MATERIALS AND METHODS}

Melting points were determined on a heating plate microscope (not corrected). IR spectra were determined on $\mathrm{KBr}$ pellets with a $400-4000 \mathrm{~cm}^{-1}$ Perkin-Elmer 16PC FTIR spectrometer. 'H-NMR spectra were determined on a Varian 300CXP apparatus (chemical shifts are expressed as $\delta$ values relative to $\mathrm{Me}_{4} \mathrm{Si}$ as standard). Elemental analysis were within $\pm 0.4 \%$ of the theoretical values calculated for the proposed formulae using a Carlo Erba Instrument CHNS Elemental Analyser. Model 1106. All reactions were monitored by thin-layer chromatography (TLC) using $0.25-\mathrm{mm}$ precoated silica gel plates (E. Merck). Preparative HPLC was performed on a Dynamax$60 \mathrm{~A}$ column $(25 \times 250 \mathrm{~mm})$, with a Beckman EM-1760 instrument. The detection wavelength was $254 \mathrm{~nm}$. Sulfonyl halides, aminoguanidine, triethylamine, carbodimides, amino acids, Cbz-amino acids, dipeptides, GABA (?-aminobutyric acid), and tosyl isocyanate used in the syntheses were commercially available compounds (from Sigma, Acros or Aldrich). Pyrylium salts used in the syntheses were prepared as described previously. ${ }^{17 a-c}$ 
Acetonitrile, acetone, dioxane, ethyl acetate (E. Merck, Darmstadt, Germany) or other solvents used in the synthesis were doubly distilled and kept on molecular sieves in order to maintain them in anhydrous conditions. Inogatran was from Astra Hassle (Molndal, Sweden). Benzamidine, NAPAP, human thrombin, human trypsin and Chromozym TH were from Sigma Chem. Co. (St. Louis, MO, USA).

\section{General Procedure for the Preparation of Compounds A1-A32}

\section{Methods $A$ and $B$}

An amount of $10 \mathrm{mM}$ sulfonyl halide (chloride for Method A, and fluoride for Method B) was dissolved in $50 \mathrm{~mL}$ of acetone and the stoichiometric amount of aminoguanidine bicarbonate $(1.36 \mathrm{~g}=10 \mathrm{mM})$ dissolved in $5 \mathrm{~mL}$ of water was added dropwise, together with the stoichiometric amount of solid $\mathrm{NaHCO}_{3}$ needed for the neutralization of the acid formed in the reaction. ${ }^{17 \mathrm{~d}, \mathrm{e}-19}$ The mixture was magnetically stirred at $25^{\circ} \mathrm{C}$ for $5 \mathrm{~h}$ when the reaction mixture was acidified with $0.1 \mathrm{~N} \mathrm{HCl}$ solution to $\mathrm{pH} 4.5$. The obtained sulfonyl derivatives, generally precipitated by leaving the above mentioned reaction mixture at $4^{\circ} \mathrm{C}$ overnight, were then filtered and recrystallized from ethanol.

\section{Method C}

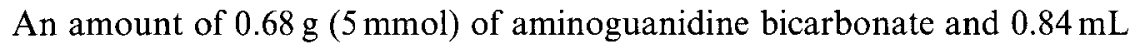
$(5 \mathrm{mmol})$ of triflic anhydride were suspended in $10 \mathrm{~mL}$ of acetone and $0.35 \mathrm{~mL}(5 \mathrm{mmol})$ of triethylamine was added dropwise. ${ }^{17-19}$ The mixture was magnetically stirred at $4^{\circ} \mathrm{C}$ for $5 \mathrm{~h}$. The solvent was then evaporated in vacuo, and the tan residue treated with $5 \mathrm{~mL}$ of cold water and acidified with $0.1 \mathrm{~N} \mathrm{HCl}$ solution. The triflate/hydrochloride salts of triethylamine being water soluble were thus separated from the triflated aminoguanidine (much less water soluble) by simple filtration. The latter compound was recrystallized from iso-propanol.

\section{Method D}

An amount of $1.36 \mathrm{~g}(10 \mathrm{mmol})$ of aminoguanidine bicarbonate, $0.70 \mathrm{~mL}$ $(10 \mathrm{mmol})$ of triethylamine and $10 \mathrm{mmol}$ of sulfobenzoic cyclic anhydride or tetrabromo- $O$-sulfobenzoic cyclic anhydride were heated at reflux in $50 \mathrm{~mL}$ of anhydrous acetonitrile for $2 \mathrm{~h} .{ }^{19-21}$ After evaporation of the solvent, the 
products were treated with $10 \mathrm{~mL}$ of water, acidified with $0.1 \mathrm{~N} \mathrm{HCl}$ solution to $\mathrm{pH} 4$, and the precipitated derivatives filtered, dried and recrystallized from ethanol.

\section{General Procedure for the Preparation of $\boldsymbol{N}$-Tosylureido Protected Amino Acids/Dipeptides}

An amount of $10 \mathrm{mM}$ of amino acid/dipeptide was suspended/dissolved in $50 \mathrm{~mL}$ of anhydrous acetone and $1.97 \mathrm{~g}(1.52 \mathrm{~mL}, 10 \mathrm{mM})$ of tosyl isocyanate was added dropwise. ${ }^{21}$ The reaction mixture was stirred at $4{ }^{\circ} \mathrm{C}$ for $4 \mathrm{~h}$, when, by means of TLC, it was observed that reaction was complete. Evaporation of the solvent in vacuo afforded white foams of $N$-tosylureido protected amino acid derivatives, which were recrystallized from ethanolwater $(1: 1, \mathrm{v} / \mathrm{v})$.

\section{General Procedure for the Preparation of Compounds A33-A40}

An amount of $1 \mathrm{mM}$ of $N$-Cbz- or $N$-tosylureido-protected amino acid/ dipeptide 7 was dissolved/suspended in $25 \mathrm{~mL}$ of anhydrous acetonitrile, and treated with $190 \mathrm{mg}(1 \mathrm{mM})$ of EDCI $\cdot \mathrm{HCl}$ and $15 \mu \mathrm{L}(1 \mathrm{mM})$ of triethylamine. ${ }^{22}$ The mixture was stirred at $4^{\circ} \mathrm{C}$ for $1 \mathrm{~h}$ to allow the formation of the mixed-anhydride type activated amino acyl compound, then $175 \mathrm{mg}(1 \mathrm{mM})$ of sulfanilyl fluoride 6 was added in three equal portions over a period of $1.5 \mathrm{~h}$. The reaction mixture was magnetically stirred at $4{ }^{\circ} \mathrm{C}$ for $16-20 \mathrm{~h}$ (TLC control), till all the amino acid dipeptide was converted to the intermediates 8 (which were not isolated). Then an amount of $136 \mathrm{mg}(1 \mathrm{mM})$ of aminoguanidine bicarbonate and $15 \mu \mathrm{L}(1 \mathrm{mM})$ of triethylamine were added in the above reaction mixture and stirring continued for $2-4 \mathrm{~h}$ at room temperature. The solvent was evaporated in vacuo and the residue taken up in ethyl acetate $(5 \mathrm{~mL})$, poured into a $5 \%$ solution of sodium bicarbonate $(5 \mathrm{~mL})$ and extracted with ethyl acetate. The combined organic layers were dried over sodium sulfate, filtered and the solvent removed in racuo. Preparative HPLC (Dynamax-60A column $(25 \times 250 \mathrm{~mm} ; 90 \%$ acetonitrile $8 \%$ methanol $2 \%$ water; flow rate of $30 \mathrm{~mL} / \mathrm{min}$ ) afforded the pure compounds $\mathbf{A 3 3}-\mathbf{A 4 0}$ as colorless solids.

\section{General Procedure for the Preparation of Derivatives 9}

An amount of $10 \mathrm{mM}$ of GABA was dissolved in $50 \mathrm{~mL}$ of anhydrous acetonitrile and the stoichiometric amount $(10 \mathrm{mM})$ of pyrylium salt and triethyl 
amine $(10 \mathrm{mM}, 1.47 \mathrm{~mL})$ were added. The reaction mixture was heated at reflux for $4-6 \mathrm{~h}$, then $2.5 \mathrm{~mL}$ of glacial acetic acid was added and refluxing continued for another $2 \mathrm{~h}$. The reaction mixture was then treated with $100 \mathrm{~mL}$ of diethylether and left overnight. The precipitate formed was filtered and recrystallized from iso-propanol.

\section{General Procedure for the Preparation of Compounds B(1-16)}

An amount of $1 \mathrm{mM}$ of pyridinium-GABA derivative 9 was dissolved/ suspended in $25 \mathrm{~mL}$ of anhydrous acetonitrile or acetone, and then treated with $224 \mathrm{mg}(1 \mathrm{mM})$ of sulfanilyl-aminoguanidine A14 and $190 \mathrm{mg}(1 \mathrm{mM})$ of $\mathrm{EDCl} \cdot \mathrm{HCl}$ or di-isopropyl-carbodiimide. The reaction mixture was magnetically stirred at $4^{\circ} \mathrm{C}$ for $15 \mathrm{~min}$, then $30 \mu \mathrm{L}(2 \mathrm{mM})$ of triethylamine was added and stirring continued for $16 \mathrm{~h}$ at $2-4^{\circ} \mathrm{C}$. Under these conditions acylation occurred only on the 4-amino group, and not on the aminoguanidine nitrogen atoms. When the reaction was carried out at room (or higher) temperature, complex reactions mixtures were obtained, containing two or three different acylation products, which could be separated by means of preparative HPLC. As only the N-4-acylated compounds were of interest, the procedure followed always involved the reaction at low temperatures, i.e., $2-4^{\circ} \mathrm{C}$. The reaction mixture was then evaporated in vacuo and the residue taken up in ethyl acetate $(5 \mathrm{~mL})$, poured into a $5 \%$ solution of sodium bicarbonate $(5 \mathrm{~mL})$ and extracted with ethyl acetate. The combined organic layers were dried over sodium sulfate and filtered, and the solvent removed in vacuo. Preparative HPLC (Dynamax-60A column, $25 \times$ $250 \mathrm{~mm} ; 88 \%$ acetonitrile $/ 9 \%$ methanol $/ 3 \%$ water; flow rate of $30 \mathrm{~mL} / \mathrm{min}$ ) afforded the pure compounds $\mathbf{B}(\mathbf{1 - 1 6 )}$ as colorless solids.

$N, N$-Dimethylsulfonyl-aminoguanidine, A1 As colorless crystals, m.p. 254-5 ${ }^{\circ} \mathrm{C}$. IR ( $\left.\mathrm{KBr}\right), \mathrm{cm}^{-1}: 1120\left(\mathrm{SO}_{2}^{\text {sym }}\right), 1190$ (imide III), $1339\left(\mathrm{SO}_{2}^{\mathrm{as}}\right), 1580$ (imide II), 1673 (imide I), 3360 br (NH, $\mathrm{NH}_{2}$ ); ${ }^{1} \mathrm{H}-\mathrm{NMR}$ (DMSO-d 6 ), $\delta$, ppm: $4.80\left(\mathrm{~s}, 6 \mathrm{H}, \mathrm{Me}_{2} \mathrm{~N}\right), 8.85$ (br s, $\left.5 \mathrm{H}, \mathrm{SO}_{2} \mathrm{NHNHC}(=\mathrm{NH}) \mathrm{NH}_{2}\right)$. Found, C, 30.09; $\mathrm{H}, 5.60 ; \mathrm{N}, 31.75 . \mathrm{C}_{3} \mathrm{H}_{11} \mathrm{~N}_{5} \mathrm{O}_{2} \mathrm{~S}$ requires: $\mathrm{C}, 30.37 ; \mathrm{H}, 5.35 ; \mathrm{N}$, $31.88 \%$.

Phenylmethylsulfonyl-aminoguanidine, A2 As colorless crystals, m.p. 264-6 ${ }^{\circ} \mathrm{C}$. IR (KBr), cm ${ }^{-1}$ : $1176\left(\mathrm{SO}_{2}^{\text {sym }}\right), 1190$ (imide III), $1365\left(\mathrm{SO}_{2}^{\text {as }}\right), 1580$ (imide II), 1673 (imide I), 3360 br (NH, $\mathrm{NH}_{2}$ ); ${ }^{1} \mathrm{H}-\mathrm{NMR}$ (DMSO-d $\mathrm{d}_{6}$ ), $\delta$, ppm: 3.23 (s, $2 \mathrm{H}, \mathrm{PhCH}$ ), 7.15-7.59 (m, 5H, ArH from $\mathrm{Ph}$ ), 9.29 (br s, $5 \mathrm{H}$, $\left.\mathrm{SO}_{2} \mathrm{NHNHC}(=\mathrm{NH}) \mathrm{NH}_{2}\right)$. Found, $\mathrm{C}, 42.15 ; \mathrm{H}, 5.03 ; \mathrm{N}, 24.20 . \mathrm{C}_{8} \mathrm{H}_{12} \mathrm{~N}_{4^{-}}$ $\mathrm{O}_{2}$ S requires: $\mathrm{C}, 42.09 ; \mathrm{H}, 5.30 ; \mathrm{N}, 24.54 \%$. 
Trifluoromethy/sulfony-aminoguanidine, A3 As colorless crystals, m.p. $247-9^{\circ} \mathrm{C}$. IR (KBr), cm ${ }^{-1}: 1169\left(\mathrm{SO}_{2}^{\mathrm{sym}}\right), 1190$ (imide III), $1340\left(\mathrm{SO}_{2}^{\text {as }}\right), 1580$ (imide II), 1673 (imide 1), 3360 br $\left(\mathrm{NH}, \mathrm{NH}_{2}\right)$ : ${ }^{1} \mathrm{H}-\mathrm{NMR}$ (DMSO-d $\left.\mathrm{d}_{6}\right), \delta$,

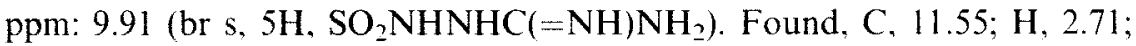
$\mathrm{N}, 27.03 . \mathrm{C}_{2} \mathrm{H}_{5} \mathrm{~F}_{3} \mathrm{~N}_{4} \mathrm{O}_{2} \mathrm{~S}$ requires: $\mathrm{C}, 11.65 ; \mathrm{H}, 2.44 ; \mathrm{N}, 27.18 \%$.

4-Fluorophenylsulfony-aminoguamidine, A4 As colorless crystals, m.p. 235-6 C. IR (KBr), cm ${ }^{-1}: 1171\left(\mathrm{SO}_{2}^{\mathrm{sym}}\right), 1190$ (imide III), $1360\left(\mathrm{SO}_{2}^{\text {as }}\right), 1580$ (imide II), 1673 (imide I), 3360 br $\left(\mathrm{NH}, \mathrm{NH}_{2}\right.$ ); ${ }^{1} \mathrm{H}-\mathrm{NMR}$ (DMSO-d ${ }_{6}$ ), $\delta$, ppm: 7.11-7.49 (m, $\mathrm{AA}^{\prime} \mathrm{BB}^{\prime}, J_{\mathrm{AB}}=7.4 \mathrm{~Hz}, 4 \mathrm{H}$. ArH, p-F-phenylene), 9.35 (br s. $5 \mathrm{H}, \mathrm{SO}_{2} \mathrm{NHNHC}(=\mathrm{NH}) \mathrm{NH}_{2}$ ). Found, C, 36.51; H, 4.09; N, 23.98. $\mathrm{C}_{7} \mathrm{H}_{9} \mathrm{FN}_{4} \mathrm{O}_{2} \mathrm{~S}$ requires: C. 36.20; H. 3.91; N. 24.12\%.

4-Chlorophenylsulfonyl-aminoguanidine, A5 As colorless crystals, m.p. $250-2{ }^{\circ} \mathrm{C}$. IR ( $\left.\mathrm{KBr}\right), \mathrm{cm}^{-1}: 1175\left(\mathrm{SO}_{2}^{\mathrm{sym}}\right), 1190$ (imide III), $1366\left(\mathrm{SO}_{2}^{\mathrm{as}}\right), 1580$ (imide II), 1673 (imide I), 3360 br (NH, NH $)_{2}$ : ${ }^{1} \mathrm{H}-\mathrm{NMR}\left(\mathrm{DMSO}-\mathrm{d}_{6}\right), \delta$, ppm: $7.10-7.56\left(\mathrm{~m} . \mathrm{AA}^{\prime} \mathrm{BB}^{\prime}, J_{\mathrm{AB}}=7.4 \mathrm{~Hz}, 4 \mathrm{H}, \mathrm{ArH}, p\right.$-Cl-phenylene), 9.33 (br s, $5 \mathrm{H}, \mathrm{SO}_{2} \mathrm{NHNHC}(=\mathrm{NH}) \mathrm{NH}_{2}$ ). Found, C. 33.89; H, 3.60; N, 22.40. $\mathrm{C}_{7} \mathrm{H}_{9} \mathrm{ClN}_{4} \mathrm{O}_{2} \mathrm{~S}$ requires: $\mathrm{C}, 33.81 ; \mathrm{H}, 3.65 ; \mathrm{N}, 22.53 \%$.

4-Bromophenylsulfony/-aminoguanidine, $\mathbf{A 6}$ As colorless crystals, m.p. $271-2^{\circ} \mathrm{C}$. IR ( $\left.\mathrm{KBr}\right), \mathrm{cm}^{-1}: 1179\left(\mathrm{SO}_{2}^{\mathrm{sym}}\right), 1190$ (imide III), $1370\left(\mathrm{SO}_{2}^{\mathrm{as}}\right), 1580$ (imide II), 1673 (imide I), 3360 br (NH. $\mathrm{NH}_{2}$ ); ' $\mathrm{H}-\mathrm{NMR}$ (DMSO-d 6 ), $\delta$, ppm: $7.15-7.47$ (m. AA'BB' $J_{\mathrm{AB}}=7.4 \mathrm{~Hz}, 4 \mathrm{H}, \mathrm{ArH}, p$-Br-phenylene), 9.28 (br s, $5 \mathrm{H}, \mathrm{SO}_{2} \mathrm{NHNHC}(=\mathrm{NH}) \mathrm{NH}_{2}$ ). Found, C. 28.75; H, 3.11; N, 19.03. $\mathrm{C}_{7} \mathrm{H}_{9} \mathrm{BrN}_{4} \mathrm{O}_{2} \mathrm{~S}$ requires: $\mathrm{C}, 28.68 ; \mathrm{H}, 3.09 ; \mathrm{N}, 19.11 \%$.

4-Iodophenylsulfonyl-aminoguanidine, A7 As colorless crystals, m.p. $218-20^{\circ} \mathrm{C}$. IR ( $\left.\mathrm{KBr}\right), \mathrm{cm}^{-1}: 1185\left(\mathrm{SO}_{2}^{\mathrm{sym}}\right), 1190$ (imide III), $1377\left(\mathrm{SO}_{2}^{\text {as }}\right), 1580$ (imide II), 1673 (imide I), 3360 br (NH, NH ${ }_{2}$ ): ${ }^{1} \mathrm{H}-\mathrm{NMR}\left(\mathrm{DMSO}_{6} \mathrm{~d}_{6}\right), \delta$, ppm: $7.10-7.50\left(\mathrm{~m}, \mathrm{AA}^{\prime} \mathrm{BB}^{\prime}, J_{\mathrm{AB}}=7.5 \mathrm{~Hz}, 4 \mathrm{H}, \mathrm{ArH}, p\right.$-I-phenylene $), 9.31$ (br s, 5H, $\mathrm{SO}_{2} \mathrm{NHNHC}(=\mathrm{NH}) \mathrm{NH}_{2}$ ). Found, C, 24.74; H, 2.95; N, 16.40. $\mathrm{C}_{7} \mathrm{H}_{9} \mathrm{IN}_{4} \mathrm{O}_{2} \mathrm{~S}$ requires: $\mathrm{C}, 24.72 ; \mathrm{H} \mathrm{2.67;} \mathrm{N,} 16.47 \%$.

4-Toluenesulfony/-aminoguanidine, $\mathbf{A 8}$ As colorless crystals, m.p. 291$3^{\circ} \mathrm{C}$. IR $(\mathrm{KBr}), \mathrm{cm}^{-1}: 1115\left(\mathrm{SO}_{2}^{\text {sym }}\right), 1190$ (imide III), $1350\left(\mathrm{SO}_{2}^{\mathrm{as}}\right), 1580$ (imide II), 1673 (imide I), 3360 br (NH, NH 2 ); ${ }^{1} \mathrm{H}-\mathrm{NMR}$ (DMSO-d $\mathrm{D}_{6}$ ), $\delta$, ppm: 2.50 (s, 3H, Me), 7.30-8.10 (m, $\mathrm{AA}^{\prime} \mathrm{BB}^{\prime}, J_{\mathrm{AB}}=7.4 \mathrm{~Hz}, 4 \mathrm{H}, \mathrm{ArH}$,

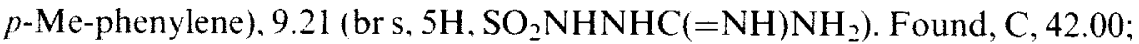
H, 5.51; N, 24.47. $\mathrm{C}_{8} \mathrm{H}_{12} \mathrm{~N}_{4} \mathrm{O}_{2} \mathrm{~S}$ requires: C, 42.09; $\mathrm{H}, 5.30 ; \mathrm{N}, 24.54 \%$.

4-Nitrophenylsulfony-aminoguanidine, A9 As yellow crystals, m.p. 269$70^{\circ} \mathrm{C}$. IR ( $\left.\mathrm{KBr}\right), \mathrm{cm}^{-1}: 1150\left(\mathrm{SO}_{2}^{\mathrm{sym}}\right), 1190$ (imide III), $1340\left(\mathrm{NO}_{2}\right), 1365$ $\left(\mathrm{SO}_{2}^{\mathrm{as}}\right), 1510\left(\mathrm{NO}_{2}\right), 1580$ (imide II), 1675 (imide I), 3360 br $\left(\mathrm{NH}, \mathrm{NH}_{2}\right) ;{ }^{1} \mathrm{H}$ NMR (DMSO-d $\left.\mathrm{d}_{6}\right), \delta, \mathrm{ppm}: 7.08-7.89\left(\mathrm{~m}, \mathrm{AA}^{\prime} \mathrm{BB}^{\prime}, J_{\mathrm{AB}}=7.4 \mathrm{~Hz}, 4 \mathrm{H}, \mathrm{ArH}\right.$, $p$ - $\mathrm{O}_{2} \mathrm{~N}$-phenylene), 9.54 (br s, $\left.5 \mathrm{H}, \quad \mathrm{SO}_{2} \mathrm{NHNHC}(=\mathrm{NH}) \mathrm{NH}_{2}\right)$ ). Found, 
$\mathrm{C}, 32.45 ; \mathrm{H}, 3.54 ; \mathrm{N}, 27.00 . \mathrm{C}_{7} \mathrm{H}_{9} \mathrm{~N}_{5} \mathrm{O}_{4} \mathrm{~S}$ requires: $\mathrm{C}, 32.43 ; \mathrm{H}, 3.50 ; \mathrm{N}$, $27.01 \%$.

3-Nitrophenylsulfonyl-aminoguanidine, $\mathbf{A 1 0}$ As yellow crystals, m.p. $262-3^{\circ} \mathrm{C}$. IR ( $\left.\mathrm{KBr}\right), \mathrm{cm}^{-1}: 1150\left(\mathrm{SO}_{2}^{\text {sym }}\right), 1190$ (imide III), $1340\left(\mathrm{NO}_{2}\right), 1375$ $\left(\mathrm{SO}_{2}^{\text {as }}\right), 1515\left(\mathrm{NO}_{2}\right), 1580$ (imide II), 1673 (imide I), 3360 br $\left(\mathrm{NH}, \mathrm{NH}_{2}\right) ;{ }^{1} \mathrm{H}$ NMR (DMSO-d $\left.\mathrm{d}_{6}\right), \delta$, ppm: 7.08-7.70 (m, 4H, ArH, $m$ - $\mathrm{O}_{2} \mathrm{~N}$-phenylene), 9.55 (br s, $\left.5 \mathrm{H}, \mathrm{SO}_{2} \mathrm{NHNHC}(=\mathrm{NH}) \mathrm{NH}_{2}\right)$. Found, C, 32.69; H, 3.27; N, 26.93. $\mathrm{C}_{7} \mathrm{H}_{9} \mathrm{~N}_{5} \mathrm{O}_{4} \mathrm{~S}$ requires: $\mathrm{C}, 32.43 ; \mathrm{H}, 3.50 ; \mathrm{N}, 27.01 \%$.

2-Nitrophenylsulfonyl-aminoguanidine, A11 As yellow crystals, m.p. 246$7^{\circ} \mathrm{C}$. IR (K Br), $\mathrm{cm}^{-1}: 1170\left(\mathrm{SO}_{2}^{\mathrm{sym}}\right), 1190$ (imide III), $1335\left(\mathrm{NO}_{2}\right), 1382\left(\mathrm{SO}_{2}^{\mathrm{as}}\right)$, $1510\left(\mathrm{NO}_{2}\right), 1580$ (imide II), 1673 (imide I), 3360 br $\left(\mathrm{NH}, \mathrm{NH}_{2}\right) ;{ }^{1} \mathrm{H}-\mathrm{NMR}$ (DMSO-d $\mathrm{d}_{6}$ ), $\delta$, ppm: 7.12-7.58(m, $4 \mathrm{H}, \mathrm{ArH}, o-\mathrm{O}_{2} \mathrm{~N}$-phenylene), 9.50 (br s, $5 \mathrm{H}$, $\left.\mathrm{SO}_{2} \mathrm{NHNHC}(=\mathrm{NH}) \mathrm{NH}_{2}\right)$. Found, $\mathrm{C}, 32.70 ; \mathrm{H}, 3.22 ; \mathrm{N}, 26.85 . \mathrm{C}_{7} \mathrm{H}_{9} \mathrm{~N}_{5} \mathrm{O}_{4} \mathrm{~S}$ requires: $\mathrm{C}, 32.43 ; \mathrm{H}, 3.50 ; \mathrm{N}, 27.01 \%$.

4-Nitro-3-chlorophenylsulfonyl-aminoguanidine, A12 As yellow crystals, m.p. $288-9^{\circ} \mathrm{C}$. IR ( $\left.\mathrm{KBr}\right), \mathrm{cm}^{-1}: 1155\left(\mathrm{SO}_{2}^{\text {sym }}\right), 1190$ (imide III), $1348\left(\mathrm{SO}_{2}^{\text {as }}\right)$, 1580 (imide II), 1673 (imide I), 3360 br $\left(\mathrm{NH}, \mathrm{NH}_{2}\right) ;{ }^{1} \mathrm{H}-\mathrm{NMR}$ (DMSO-d $\mathrm{d}_{6}$ ), $\delta$, ppm: $7.09-7.69$ (m, 3H, $\mathrm{ArH}), 9.15$ (br s, $5 \mathrm{H}, \mathrm{SO}_{2} \mathrm{NHNHC}(=\mathrm{NH}) \mathrm{NH}_{2}$ ). Found, $\mathrm{C}, 28.96 ; \mathrm{H}, 2.72 ; \mathrm{N}, 23.80 . \mathrm{C}_{7} \mathrm{H}_{8} \mathrm{ClN}_{5} \mathrm{O}_{4} \mathrm{~S}$ requires: $\mathrm{C}, 28.63 ; \mathrm{H}$, $2.75 ; \mathrm{N}, 23.85 \%$.

4-Acetylaminophenylsulfonyl-aminoguanidine, A13 As colorless crystals, m.p. 237-9 ${ }^{\circ} \mathrm{C}$. IR ( $\left.\mathrm{KBr}\right), \mathrm{cm}^{-1}: 1155\left(\mathrm{SO}_{2}^{\text {sym }}\right), 1190$ (imide III), $1350\left(\mathrm{SO}_{2}^{\text {as }}\right)$, 1530 (amide II), 1580 (imide II), 1670 (imide I), 1688 (amide I), $3360 \mathrm{br}$ $\left(\mathrm{NH}, \mathrm{NH}_{2}\right.$ ); ${ }^{1} \mathrm{H}-\mathrm{NMR}$ (DMSO-d $\mathrm{d}_{6}$ ), $\delta$, ppm: 1.80 (s, 3H, Me from Ac), 6.21 (s, $1 \mathrm{H}, \mathrm{Ac} N H), 7.07-7.80\left(\mathrm{~m}, \mathrm{AA}^{\prime} \mathrm{BB}^{\prime}, J_{\mathrm{AB}}=7.4 \mathrm{~Hz}, 4 \mathrm{H}, \mathrm{ArH}, p-\mathrm{AcNH}-\right.$ phenylene), 9.13 (br s, $5 \mathrm{H}, \mathrm{SO}_{2} \mathrm{NHNHC}(=\mathrm{NH}) \mathrm{NH}_{2}$ ). Found, $\mathrm{C}, 39.81 ; \mathrm{H}$, 4.65; N, 25.77. $\mathrm{C}_{9} \mathrm{H}_{13} \mathrm{~N}_{5} \mathrm{O}_{3} \mathrm{~S}$ requires: $\mathrm{C}, 39.85 ; \mathrm{H}, 4.83 ; \mathrm{N}, 25.81 \%$.

4-Aminophenylsulfonyl-aminoguanidine, $\mathbf{A 1 4}$ As colorless crystals, m.p. 215-6 ${ }^{\circ} \mathrm{C}$ (lit. [19] m.p. $209-10^{\circ} \mathrm{C}$ ). IR ( $\left.\mathrm{KBr}\right), \mathrm{cm}^{-1}: 1155$ ( $\left.\mathrm{SO}_{2}^{\text {sym }}\right), 1190$ (imide III), 1354 ( $\left.\mathrm{SO}_{2}^{\mathrm{as}}\right), 1580$ (imide II), 1675 (imide I), $3360 \mathrm{br}\left(\mathrm{NH}, \mathrm{NH}_{2}\right.$ ); ${ }^{1} \mathrm{H}-\mathrm{NMR}$ (DMSO-d $\mathrm{d}_{6}$ ), $\delta$, ppm: 5.46 (s, 2H, $\mathrm{H}_{2} \mathrm{~N}$-phenylene), 7.05-7.65 (m, $\mathrm{AA}^{\prime} \mathrm{BB}^{\prime}, J_{\mathrm{AB}}=7.4 \mathrm{~Hz}, 4 \mathrm{H}, \mathrm{ArH}, p-\mathrm{H}_{2} \mathrm{~N}$-phenylene), 9.21 (br s, $5 \mathrm{H}, \mathrm{SO}_{2^{-}}$ $\left.\mathrm{NHNHC}(=\mathrm{NH}) \mathrm{NH}_{2}\right), 10.23(\mathrm{~s}, 1 \mathrm{H}, \mathrm{OH})$. Found, C, 36.52; H, 4.75; N, 30.47. $\mathrm{C}_{7} \mathrm{H}_{11} \mathrm{~N}_{5} \mathrm{O}_{2} \mathrm{~S}$ requires: $\mathrm{C}, 36.67 ; \mathrm{H}, 4.84 ; \mathrm{N}, 30.55 \%$. This compound has also been prepared by deacetylation in acidic medium of A13, as reported by Winnek et al. ${ }^{19}$

3-Aminophenylsulfonyl-aminoguanidine, A15 As tan crystals, m.p. 205$6^{\circ} \mathrm{C}$. IR $(\mathrm{KBr}), \mathrm{cm}^{-1}: 1172\left(\mathrm{SO}_{2}^{\text {sym }}\right), 1190$ (imide III), $1360\left(\mathrm{SO}_{2}^{\mathrm{as}}\right), 1580$ (imide II), 1673 (imide I), $3360 \mathrm{br}\left(\mathrm{NH}, \mathrm{NH}_{2}\right.$ ); ${ }^{1} \mathrm{H}-\mathrm{NMR}$ (DMSO-d $\mathrm{d}_{6}$ ), $\delta$, ppm: $5.22\left(\mathrm{~s}, 2 \mathrm{H}, \mathrm{H}_{2} \mathrm{~N}\right.$-phenylene), $7.21-7.59$ (m, $4 \mathrm{H}, \mathrm{ArH}, m-\mathrm{H}_{2} \mathrm{~N}$-phenylene), 
9.20 (br s, $5 \mathrm{H}, \mathrm{SO}_{2} \mathrm{NHNHC}(=\mathrm{NH}) \mathrm{NH}_{2}$ ). Found, C, 36.71; H, 4.93; N, 30.52. $\mathrm{C}_{7} \mathrm{H}_{1} \mathrm{~N}_{5} \mathrm{O}_{2} \mathrm{~S}$ requires: $\mathrm{C}, 36.67 ; \mathrm{H}, 4.84 ; \mathrm{N}, 30.55 \%$.

Pentafluorophenylsulfonyl-aminoguanidine, A16 As colorless crystals, m.p. $198-200^{\circ} \mathrm{C}$. IR $(\mathrm{KBr}), \mathrm{cm}^{-1}: 1148\left(\mathrm{SO}_{2}^{\mathrm{sym}}\right), 1336\left(\mathrm{SO}_{2}^{\mathrm{as}}\right), 1190$ (imide III), 1580 (imide II), 1673 (imide I), 3360 br (NH, NH ${ }_{2}$ ); ${ }^{1} \mathrm{H}-\mathrm{NMR}$ (DMSO$\mathrm{d}_{6}$ ), $\delta$, ppm: 10.10 (br s, $5 \mathrm{H}, \mathrm{SO}_{2} \mathrm{NHNHC}(=\mathrm{NH}) \mathrm{NH}_{2}$ ). Found, $\mathrm{C}, 27.92 ; \mathrm{H}$, 1.45; N, 18.40. $\mathrm{C}_{7} \mathrm{H}_{5} \mathrm{~F}_{5} \mathrm{~N}_{4} \mathrm{O}_{2} \mathrm{~S}$ requires: C. 27.64; $\mathrm{H}, 1.66 ; \mathrm{N}, 18.42 \%$.

2-Carboxyphenylsulfonl-aminoguanidine, A17 As colorless crystals, m.p. $271-2^{\circ} \mathrm{C}$. IR (KBr), cm ${ }^{-1}: 1153\left(\mathrm{SO}_{2}^{\mathrm{sym}}\right), 1190$ (imide III), $1354\left(\mathrm{SO}_{2}^{\mathrm{as}}\right)$, 1580 (imide II), 1673 (imide I), 1722 (COOH), 3360 br (NH, $\mathrm{NH}_{2}$ ); ${ }^{1} \mathrm{H}-\mathrm{NMR}$ (DMSO-d ${ }_{6}$ ), $\delta, \mathrm{ppm}: 7.15-7.62$ (m, 4H, ArH, o-HOOC-phenylene), 9.80 (br s. $\left.5 \mathrm{H} . \mathrm{SO}_{2} \mathrm{NHNHC}(=\mathrm{NH}) \mathrm{NH}_{2}\right), 10.35$ (br s, $\left.1 \mathrm{H}, \mathrm{COOH}\right)$. Found, C, 37.54; $\mathrm{H}, 3.58 ; \mathrm{N}, 21.55 . \mathrm{C}_{8} \mathrm{H}_{10} \mathrm{~N}_{4} \mathrm{O}_{4} \mathrm{~S}$ requires: C, 37.21; $\mathrm{H}, 3.90 ; \mathrm{N}, 21.69 \%$.

3-Carboxyphenylsulfonyl-aminoguanidine, A18 As colorless crystals, m.p. $248-9^{\circ} \mathrm{C}$. IR (KBr), $\mathrm{cm}^{-1}: 1136\left(\mathrm{SO}_{2}^{\mathrm{sym}}\right), 1190$ (imide III), $1349\left(\mathrm{SO}_{2}^{\text {as }}\right)$, 1580 (imide Il), 1673 (imide I), $1720(\mathrm{COOH}), 3360$ br (NH, $\mathrm{NH}_{2}$ ); ${ }^{1} \mathrm{H}-\mathrm{NMR}$ (DMSO-d ${ }_{6}$ ), $\delta$, ppm: 7.09-7.69 (m, 4H, ArH, m-HOOC-phenylene), 9.64 (br $\left.\mathrm{s}, 5 \mathrm{H}, \mathrm{SO}_{2} \mathrm{NHNHC}(=\mathrm{NH}) \mathrm{NH}_{2}\right), 10.21$ (br s, $\left.1 \mathrm{H}, \mathrm{COOH}\right)$. Found, C, 37.08; H. $3.82 ; \mathrm{N}, 21.50 . \mathrm{C}_{8} \mathrm{H}_{10} \mathrm{~N}_{4} \mathrm{O}_{4} \mathrm{~S}$ requires: C. $37.21 ; \mathrm{H}, 3.90 ; \mathrm{N}, 21.69 \%$.

4-Carboxypheny/sulfony/-aminoguanidine, A19 As colorless crystals, m.p. $283-5^{\circ}$ C. IR (KBr), cm ${ }^{1}: 1148\left(\mathrm{SO}_{2}^{\mathrm{sym}}\right), 1190$ (imide III), 1359 ( $\mathrm{SO}_{2}^{\text {as }}$ ), 1580 (imide II), 1673 (imide I), $1720(\mathrm{COOH}), 3360$ br $\left(\mathrm{NH}, \mathrm{NH}_{2}\right) ;{ }^{1} \mathrm{H}-\mathrm{NMR}$ $\left(\right.$ DMSO-d $_{6}$ ), $\delta$, ppm: $7.15-7.67\left(\mathrm{AA}^{\prime} \mathrm{BB}^{\prime}, J_{\mathrm{AB}}=7.4 \mathrm{~Hz}, 4 \mathrm{H}, \mathrm{ArH}, p\right.$-HOOCphenylene), 9.52 (br s, $5 \mathrm{H}, \mathrm{SO}_{2} \mathrm{NHNHC}(=\mathrm{NH}) \mathrm{NH}_{2}$ ), 10.24 (br s, $1 \mathrm{H}$, $\mathrm{COOH}$ ). Found, C, 37.23; H. 3.64; N. 21.62. $\mathrm{C}_{8} \mathrm{H}_{10} \mathrm{~N}_{4} \mathrm{O}_{4} \mathrm{~S}$ requires: C, 37.21; H. 3.90 ; N. $21.69 \%$.

2-Carboxytetrabromopheny/sulfonyl-aminoguanidine, $\mathbf{A 2 0}$ As colorless crystals, m.p. $211-2^{\circ} \mathrm{C}$. IR (KBr), $\mathrm{cm}^{-1}: 1155$ (SO ${ }_{2}^{\text {sym }}$ ), 1190 (imide III), 1370 $\left(\mathrm{SO}_{2}^{\mathrm{as}}\right), 1580$ (imide II), 1673 (imide I), $1720(\mathrm{COOH}), 3360$ br $\left(\mathrm{NH}, \mathrm{NH}_{2}\right)$; ${ }^{1} \mathrm{H}$-NMR (DMSO-d 6 ), $\delta$, ppm: 9.70 (br s, 5H, $\mathrm{SO}_{2} \mathrm{NHNHC}(=\mathrm{NH}) \mathrm{NH}_{2}$ ), 10.40 (br s, $1 \mathrm{H}, \mathrm{COOH}$ ). Found, C, 16.50; H, 1.40; N, 9.56. $\mathrm{C}_{8} \mathrm{H}_{6} \mathrm{Br}_{4} \mathrm{~N}_{4} \mathrm{O}_{4} \mathrm{~S}$ requires: $\dot{\mathrm{C}}, 16.74 ; \mathrm{H}, 1.05 ; \mathrm{N}, 9.76 \%$.

4-Methoxyphenylsulfonyl-aminoguanidine, A21 As colorless crystals, m.p. $249-51^{\circ} \mathrm{C}$. IR (KBr). $\mathrm{cm}^{-1}: 1169\left(\mathrm{SO}_{2}^{\text {sym }}\right), 1190$ (imide III), $1315\left(\mathrm{SO}_{2}^{\text {as }}\right)$, 1580 (imide II), 1673 (imide I), 3360 br (NH, $\mathrm{NH}_{2}$ ); ${ }^{1} \mathrm{H}-\mathrm{NMR}$ (DMSO-d 6 ), $\delta$. ppm: $3.50(\mathrm{~s}, 3 \mathrm{H}, \mathrm{MeO}), 7.10-7.83\left(\mathrm{~m}, \mathrm{AA}^{\prime} \mathrm{BB}^{\prime}, J_{\mathrm{AB}}=7.4 \mathrm{~Hz}, 4 \mathrm{H}, \mathrm{ArH}\right.$, p-MeO-phenylene), 9.06 (brs. $\left.5 \mathrm{H}, \mathrm{SO}_{2} \mathrm{NHNHC}(=\mathrm{NH}) \mathrm{NH}_{2}\right)$. Found, $\mathrm{C}, 39.56$; $\mathrm{H}, 4.88 ; \mathrm{N}, 22.73 . \mathrm{C}_{8} \mathrm{H}_{12} \mathrm{~N}_{4} \mathrm{O}_{3} \mathrm{~S}$ requires: $\mathrm{C}, 39.34 ; \mathrm{H}, 4.95 ; \mathrm{N}, 22.94 \%$.

2.4,6-Trimethylphenylsulfony-aminoguanidine, $\mathbf{A 2 2}$ As colorless crystals, m.p. $210-3^{\circ} \mathrm{C}$. IR $(\mathrm{KBr}), \mathrm{cm}^{-1}: 1170\left(\mathrm{SO}_{2}^{\text {sym }}\right), 1190$ (imide III), $1319\left(\mathrm{SO}_{2}^{\text {as }}\right)$, 
1580 (imide II), 1673 (imide I), 3360 br (NH, NH $\left.{ }_{2}\right) ;{ }^{1} \mathrm{H}-\mathrm{NMR}$ (DMSO-d $\mathrm{d}_{6}$ ), $\delta$, ppm: 2.50 (s, 3H, 4-Me), 2.75 (s, 6H, 2,6- $\mathrm{Me}_{2}$ ), 7.10-7.85 (m, 2H, ArH), 9.09 (br s, $\left.5 \mathrm{H}, \mathrm{SO}_{2} \mathrm{NHNHC}(=\mathrm{NH}) \mathrm{NH}_{2}\right)$. Found, $\mathrm{C}, 48.67 ; \mathrm{H}, 6.17 ; \mathrm{N}$, 21.75. $\mathrm{C}_{10} \mathrm{H}_{16} \mathrm{~N}_{4} \mathrm{O}_{2} \mathrm{~S}$ requires: $\mathrm{C}, 48.86 ; \mathrm{H}, 6.29 ; \mathrm{N}, 21.86 \%$.

4-Methoxy-3-aminophenylsulfonyl-aminoguanidine, $\mathbf{A 2 3}$ As colorless crystals, m.p. $233-4^{\circ} \mathrm{C}$. IR (KBr), cm ${ }^{-1}: 1160$ ( $\left.\mathrm{SO}_{2}^{\text {sym }}\right), 1190$ (imide III), 1353 $\left(\mathrm{SO}_{2}^{\text {as }}\right), 1580$ (imide II), 1673 (imide I), 3360 br $\left(\mathrm{NH}, \mathrm{NH}_{2}\right) ;{ }^{1} \mathrm{H}-\mathrm{NMR}$ (DMSO-d ${ }_{6}$ ), $\delta$, ppm: 3.50 (s, $3 \mathrm{H}, \mathrm{MeO}$ ), 5.22 (s, $2 \mathrm{H}, \mathrm{H}_{2} \mathrm{~N}$-phenylene), $7.20-$ $7.64\left(\mathrm{~m}, 3 \mathrm{H}, \mathrm{ArH}\right.$, trisubstituted-phenyl), 9.10 (br s, $5 \mathrm{H}, \mathrm{SO}_{2} \mathrm{NHNHC}-$ $\left.(=\mathrm{NH}) \mathrm{NH}_{2}\right)$. Found, $\mathrm{C}, 36.98 ; \mathrm{H}, 5.13 ; \mathrm{N}, 26.85 . \mathrm{C}_{8} \mathrm{H}_{13} \mathrm{~N}_{5} \mathrm{O}_{3} \mathrm{~S}$ requires: $\mathrm{C}$, $37.06 ; \mathrm{H}, 5.05 ; \mathrm{N}, 27.01 \%$.

2-Hydroxy-3,5-dichlorophenylsulfonyl-aminoguanidine, A24 As tan crystals, m.p. $191-2^{\circ} \mathrm{C}$. IR ( $\left.\mathrm{KBr}\right), \mathrm{cm}^{-1}: 1143\left(\mathrm{SO}_{2}^{\text {sym }}\right), 1190$ (imide III), 1339 ( $\mathrm{SO}_{2}^{\text {as }}$ ), 1580 (imide II), 1673 (imide I), $3360 \mathrm{br}\left(\mathrm{OH}, \mathrm{NH}, \mathrm{NH}_{2}\right)$; ${ }^{1} \mathrm{H}-\mathrm{NMR}$ (DMSO-d 6 ), $\delta$, ppm: 6.22 (br s, 1H, HO), 7.35 (s, 1H, ArH, 4H), $7.80(\mathrm{~s}, 1 \mathrm{H}$, $\mathrm{ArH}, 6 \mathrm{H}), 9.24$ (br s, $5 \mathrm{H}, \mathrm{SO}_{2} \mathrm{NHNHC}(=\mathrm{NH}) \mathrm{NH}_{2}$ ). Found, $\mathrm{C}, 28.30 ; \mathrm{H}$, $2.63 ; \mathrm{N}, 18.59 . \mathrm{C}_{7} \mathrm{H}_{8} \mathrm{Cl}_{2} \mathrm{~N}_{4} \mathrm{O}_{3} \mathrm{~S}$ requires: $\mathrm{C}, 28.11 ; \mathrm{H}, 2.70 ; \mathrm{N}, 18.73 \%$.

4-(4-Dimethylaminophenylazo)-benzenesulfonyl-aminoguanidine, $\mathbf{A 2 5}$ As yellow crystals, m.p. $208-9^{\circ} \mathrm{C}$. IR $(\mathrm{KBr}), \mathrm{cm}^{-1}: 1156\left(\mathrm{SO}_{2}^{\text {sym }}\right), 1190$ (imide III), 1310 ( $\mathrm{SO}_{2}^{\text {as }}$ ), 1580 (imide II), 1673 (imide I), $3360 \mathrm{br}\left(\mathrm{NH}, \mathrm{NH}_{2}\right) ;{ }^{1} \mathrm{H}-$ NMR (DMSO-d $\mathrm{d}_{6}$ ), $\delta$, ppm: $3.00\left(\mathrm{~s}, 6 \mathrm{H}, \mathrm{Me}_{2} \mathrm{~N}\right), 7.08-8.10\left(\mathrm{~m}, \mathrm{AA}^{\prime} \mathrm{BB}^{\prime}\right.$, $J_{\mathrm{AB}}=7.4 \mathrm{~Hz}, 8 \mathrm{H}, \mathrm{ArH}$ ), 9.15 (br s, $5 \mathrm{H}, \mathrm{SO}_{2} \mathrm{NHNHC}(=\mathrm{NH}) \mathrm{NH}_{2}$ ). Found, $\mathrm{C}, 49.69 ; \mathrm{H}, 5.50 ; \mathrm{N}, 27.05 . \mathrm{C}_{15} \mathrm{H}_{19} \mathrm{~N}_{7} \mathrm{O}_{2} \mathrm{~S}$ requires: $\mathrm{C}, 49.85 ; \mathrm{H}, 5.30 ; \mathrm{N}$, $27.13 \%$.

5-Dimethylamino-1-naphthalenesulfonyl-aminoguanidine, A26 As yellow crystals, m.p. $230-2^{\circ} \mathrm{C} . \mathrm{IR}(\mathrm{KBr}), \mathrm{cm}^{-1}: 1169$ ( $\left.\mathrm{SO}_{2}^{\text {sym }}\right), 1190$ (imide III), 1328 $\left(\mathrm{SO}_{2}^{\text {as }}\right), 1580$ (imide II), 1673 (imide I), 3360 br $\left(\mathrm{NH}, \mathrm{NH}_{2}\right) ;{ }^{1} \mathrm{H}-\mathrm{NMR}$ (DMSO-d $\mathrm{d}_{6}$ ), $\delta$, ppm: $3.00\left(\mathrm{~s}, 6 \mathrm{H}, \mathrm{Me}_{2} \mathrm{~N}\right), 7.66-7.80\left(\mathrm{~m}, 4 \mathrm{H}, \mathrm{H}^{2}, \mathrm{H}^{3}, \mathrm{H}^{6}, \mathrm{H}^{7}\right.$ from naphthalene), $7.96\left(\mathrm{~d}, 1 \mathrm{H}, \mathrm{H}^{4}\right.$ from the substituted-naphthyl), 8.15 $\left(\mathrm{m}, 1 \mathrm{H}, \mathrm{H}^{8}\right.$ from the substituted-naphthyl), $9.23\left(\mathrm{br} \mathrm{s}, 5 \mathrm{H}, \mathrm{SO}_{2} \mathrm{NHNHC}-\right.$ $(=\mathrm{NH}) \mathrm{NH}_{2}$ ). Found, $\mathrm{C}, 50.60 ; \mathrm{H}, 5.72 ; \mathrm{N}, 22.69 . \mathrm{C}_{13} \mathrm{H}_{17} \mathrm{~N}_{5} \mathrm{O}_{2} \mathrm{~S}$ requires: $\mathrm{C}$, $50.80 ; \mathrm{H}, 5.57 ; \mathrm{N}, 22.78 \%$.

1-Naphthalenesulfonyl-aminoguanidine, A27 As white crystals, m.p. 261- $3^{\circ} \mathrm{C}$. IR ( $\left.\mathrm{KBr}\right), \mathrm{cm}^{-1}$ : $1164\left(\mathrm{SO}_{2}^{\mathrm{sym}}\right), 1190$ (imide III), $1361\left(\mathrm{SO}_{2}^{\mathrm{as}}\right), 1580$ (imide II), 1673 (imide I), $3360 \mathrm{br}\left(\mathrm{NH}, \mathrm{NH}_{2}\right.$ ); ${ }^{1} \mathrm{H}-\mathrm{NMR}$ (DMSO-d ${ }_{6}$ ), $\delta$, ppm: 7.66-7.79 (m, 4H, $\mathrm{H}^{2}, \mathrm{H}^{3}, \mathrm{H}^{6}, \mathrm{H}^{7}$ from naphthalene), $7.94\left(\mathrm{~m}, 1 \mathrm{H}, \mathrm{H}^{5}\right.$ or $\mathrm{H}^{8}$ from the substituted-naphthyl), $7.98\left(\mathrm{~d}, 1 \mathrm{H}, \mathrm{H}^{4}\right.$ from the substitutednaphthyl), $8.16\left(\mathrm{~m}, 1 \mathrm{H}, \mathrm{H}^{5}\right.$ or $\mathrm{H}^{8}$ from the substituted-naphthyl), 9.16 (br s, $\left.5 \mathrm{H}, \mathrm{SO}_{2} \mathrm{NHNHC}(=\mathrm{NH}) \mathrm{NH}_{2}\right)$. Found, $\mathrm{C}, 49.69 ; \mathrm{H}, 4.30 ; \mathrm{N}, 21.04 . \mathrm{C}_{11} \mathrm{H}_{12^{-}}$ $\mathrm{N}_{4} \mathrm{O}_{2} \mathrm{~S}$ requires: $\mathrm{C}, 49.99 ; \mathrm{H}, 4.58 ; \mathrm{N}, 21.20 \%$. 
2-Naphthalenesulfonyl-aminoguanidine, A28 As white crystals, m.p. 260-1 ${ }^{\circ} \mathrm{C}$. IR ( $\left.\mathrm{KBr}\right), \mathrm{cm}^{-1}: 1160\left(\mathrm{SO}_{2}^{\mathrm{sym}}\right), 1190$ (imide III), $1380\left(\mathrm{SO}_{2}^{\text {as }}\right), 1580$ (imide II), 1673 (imide I), 3360 br $\left(\mathrm{NH}, \mathrm{NH}_{2}\right) ;{ }^{\mathrm{l}} \mathrm{H}-\mathrm{NMR}\left(\mathrm{DMSO}-\mathrm{d}_{6}\right), \delta$, ppm: 7.66-7.74 (m, 2H, $\mathrm{H}^{6}, \mathrm{H}^{7}$ from naphthalene), $7.94\left(\mathrm{~m}, 1 \mathrm{H}, \mathrm{H}^{5}\right.$ or $\mathrm{H}^{8}$ from the substituted-naphthyl), $7.96\left(\mathrm{~d}, 1 \mathrm{H}, \mathrm{H}^{3}\right.$ or $\mathrm{H}^{4}$ from the substitutednaphthyl), 8.20 (d, $1 \mathrm{H}, \mathrm{H}^{4}$ or $\mathrm{H}^{3}$ from the substituted-naphthyl), $8.33(\mathrm{~s}, 1 \mathrm{H}$, $\mathrm{H}^{1}$ of the substituted-naphthyl), $8.35\left(\mathrm{dd}, 9.7 \mathrm{~Hz}, 2.3,1 \mathrm{H}, \mathrm{H}^{8}\right.$ or $\mathrm{H}^{5}$ from the substituted-naphthyl), 9.21 (br s, $5 \mathrm{H}, \mathrm{SO}_{2} \mathrm{NHNHC}(=\mathrm{NH}) \mathrm{NH}_{2}$ ). Found, $\mathrm{C}$, 49.76; H, 4.54; N, 21.12. $\mathrm{C}_{11} \mathrm{H}_{12} \mathrm{~N}_{4} \mathrm{O}_{2} \mathrm{~S}$ requires: $\mathrm{C}, 49.99 ; \mathrm{H}, 4.58 ; \mathrm{N}$, $21.20 \%$.

Perfluoro-n-butylsulfonyl-aminoguanidine, A29 As colorless crystals, m.p. $122-4^{\circ} \mathrm{C}$. IR (KBr), $\mathrm{cm}^{-1}: 1170\left(\mathrm{SO}_{2}^{\text {sym }}\right), 1190$ (imide III), $1365\left(\mathrm{SO}_{2}^{\text {as }}\right)$, 1580 (imide II), 1673 (imide I), 3360 br ( $\mathrm{NH}, \mathrm{NH}_{2}$ ); ${ }^{1} \mathrm{H}-\mathrm{NMR}$ (DMSO-d 6 ), $\delta$. ppm: 9.57 (br s, $5 \mathrm{H}, \mathrm{SO}_{2} \mathrm{NHNHC}(=\mathrm{NH}) \mathrm{NH}_{2}$ ). Found, C, 16.95; H, 1.45; N. 15.66, $\mathrm{C}_{5} \mathrm{H}_{5} \mathrm{~F}_{9} \mathrm{~N}_{4} \mathrm{O}_{2} \mathrm{~S}$ requires: $\mathrm{C}, 16.86 ; \mathrm{H}, 1.42 ; \mathrm{N}, 15.73 \%$.

Perfluoro-n-octylsulfonyl-aminoguanidine, A30 As colorless crystals, m.p. $99-101^{\circ} \mathrm{C}$. IR ( $\left.\mathrm{KBr}\right), \mathrm{cm}^{-1}: 1176\left(\mathrm{SO}_{2}^{\text {sym }}\right), 1190$ (imide III), $1366\left(\mathrm{SO}_{2}^{\text {as }}\right)$, 1580 (imide II), 1673 (imide I), 3360 br (NH, $\mathrm{NH}_{2}$ ); ${ }^{1} \mathrm{H}-\mathrm{NMR}$ (DMSO-d 6 ), o. ppm: 9.43 (br s, $\left.5 \mathrm{H}, \mathrm{SO}_{2} \mathrm{NHNHC}=\mathrm{NH}\right) \mathrm{NH}_{2}$ ). Found, $\mathrm{C}, 19.61 ; \mathrm{H}, 0.80$; $\mathrm{N}, 10.05 . \mathrm{C}_{9} \mathrm{H}_{5} \mathrm{~F}_{17} \mathrm{~N}_{4} \mathrm{O}_{2} \mathrm{~S}$ requires: $\mathrm{C}, 19.44 ; \mathrm{H}, 0.91 ; \mathrm{N}, 10.07 \%$.

2-Thienylsulfonyl-aminoguanidine, A31 As colorless crystals, m.p. 240 1 C. IR (KBr) $\mathrm{cm}^{-1}: 1154\left(\mathrm{SO}_{2}^{\text {sym }}\right), 1190$ (imide III) $1350\left(\mathrm{SO}_{2}^{\text {as }}\right), 1580$ (imide II), 1673 (imide I), 3360 br (NH, NH ${ }_{2}$ ); ${ }^{1} \mathrm{H}-\mathrm{NMR}$ (DMSO-d $\mathrm{d}_{6}$ ), $\delta, \mathrm{ppm:} 6.28$ (dd, 3.8, 2.5, 1H, from thienyl), 6.88 (dd, $3.8,1.8 \mathrm{~Hz}, 1 \mathrm{H}$, from thienyl), 7.16 (dd, $2.5,1.8 \mathrm{~Hz}, 1 \mathrm{H}$, from thienyl), 9.29 (br s, $5 \mathrm{H}, \mathrm{SO}_{2} \mathrm{NHNHC}(=\mathrm{NH})-$ $\mathrm{NH}_{2}$ ). Found, $\mathrm{C}, 27.09 ; \mathrm{H}, 3.43 ; \mathrm{N}, 25.36 . \mathrm{C}_{5} \mathrm{H}_{8} \mathrm{~N}_{4} \mathrm{O}_{2} \mathrm{~S}_{2}$ requires: $\mathrm{C}, 27.26$; H. 3.66; N. 25.44\%.

10-Camphorsulfonyl-aminoguanidine, A32 As white crystals, m.p. 199$201^{\circ} \mathrm{C}$. IR $(\mathrm{KBr}), \mathrm{cm}^{-1}: 1125\left(\mathrm{SO}_{2}^{\text {sym }}\right), 1190$ (imide $\left.\mathrm{III}\right), 1376\left(\mathrm{SO}_{2}^{\mathrm{as}}\right), 1580$ (imide II), 1673 (imide I), 3360 br ( $\left.\mathrm{NH}, \mathrm{NH}_{2}\right)$; ${ }^{1} \mathrm{H}-\mathrm{NMR}\left(\mathrm{DMSO}-\mathrm{d}_{6}\right), \delta$, ppm: $1.40(\mathrm{~s}, 6 \mathrm{H}, 2 \mathrm{Me}), 2.74-2.98\left(\mathrm{~m}, 4 \mathrm{H}, \mathrm{CH}_{2} \mathrm{CH}_{2}\right), 3.15\left(\mathrm{~m}, 2 \mathrm{H}, \mathrm{CH}_{2} \mathrm{CO}\right)$, $3.72\left(\mathrm{~s}, 2 \mathrm{H}, \mathrm{CH}_{2} \mathrm{SO}_{2}\right), 4.05(\mathrm{~m}, 1 \mathrm{H}, \mathrm{CH}), 9.03$ (br s, 5H, SO $\mathrm{NHNHC}_{2}$ $\left.(=\mathrm{NH}) \mathrm{NH}_{2}\right)$. Found: $\mathrm{C}, 45.76 ; \mathrm{H}, 7.12 ; \mathrm{N}, 19.39 . \mathrm{C}_{11} \mathrm{H}_{20} \mathrm{~N}_{4} \mathrm{O}_{3} \mathrm{~S}$ requires: $\mathrm{C}$, $45.82 ;$ H. $6.99 ;$ N, $19.43 \%$.

4-( N-Benzyloxycarbonyl-D-phenylalanylamido)-benzenesulfonyl-aminoguanidine, A33 Its m.p. 250-2 ${ }^{\circ} \mathrm{C} .{ }^{1} \mathrm{H}$-NMR (DMSO-D $2 \mathrm{O}$ ), $\delta$, ppm: $3.10-$ $3.56\left(\mathrm{~m}, 2 \mathrm{H}, \mathrm{CH}_{2} \mathrm{CH}\right.$ of Phe), 4.11 (dd, ${ }^{3} J_{\mathrm{HH}}=5.0,{ }^{3} J_{\mathrm{HH}}=7.8 ; 1 \mathrm{H}, \mathrm{CH}_{2} \mathrm{CH}$ of Phe), $5.10\left(\mathrm{~s}, 2 \mathrm{H}, \mathrm{PhCH}_{2} \mathrm{O}\right), 7.20-7.42\left(\mathrm{~m}, 10 \mathrm{H}, \mathrm{H}_{\text {arom }}\right.$ of Phe and $\left.\mathrm{PhCH}_{2} \mathrm{O}\right), 7.65\left(\mathrm{~d},{ }^{3} J_{\mathrm{HH}}=7.9,2 \mathrm{H}, H_{\text {ortho }}\right.$ of XNHC $\left.6 \mathrm{H}_{4} \mathrm{SO}_{2}\right), 7.90\left(\mathrm{~d},{ }^{3} J_{\mathrm{HH}}=\right.$ 7.9. $2 \mathrm{H}, H_{\text {meta }}$ of $\left.\mathrm{XNHC}_{6} \mathrm{H}_{4} \mathrm{SO}_{2}\right), 9.32\left(\right.$ br s, $\left.5 \mathrm{H}, \mathrm{SO}_{2} \mathrm{~N} H \mathrm{NHC}(=\mathrm{N} H) \mathrm{N} H_{2}\right)$; 
${ }^{13} \mathrm{C}-\mathrm{NMR}$ (DMSO-D $2 \mathrm{O}$ ), $\delta$, ppm: 41.6 (s, $\mathrm{CH}_{2} \mathrm{CH}$ of Phe), 59.5 (s, $\mathrm{CHCH}_{2}$ of Phe), 74.2 (s, $\mathrm{PhCH}_{2} \mathrm{O}$ ), 130.9 (s, $C_{\text {para }}$ of Phe), 133.8 (s, $C_{\text {meta }}$ of XNH$\mathrm{C}_{6} \mathrm{H}_{4} \mathrm{SO}_{2}$ ), 133.9 (s, $C_{\text {meta }}$ of Phe), 134.5 (s, $C_{\text {ortho }}$ of Phe), 135.5 (s, $C_{\text {ortho }}$ of $\left.\mathrm{XNHC}_{6} \mathrm{H}_{4} \mathrm{SO}_{2}\right), 141.5\left(\mathrm{~s}, C_{\text {ipso }}\right.$ of Phe), $142.9\left(\mathrm{~s}, \mathrm{C}_{\text {para }}\right.$ of $\mathrm{XNHC}_{6} \mathrm{H}_{4} \mathrm{SO}_{2}$ ), $148.9\left(\mathrm{~s}, C_{\text {ipso }}\right.$ of $\left.\mathrm{XNHC}_{6} \mathrm{H}_{4} \mathrm{SO}_{2}\right), 173.0(\mathrm{~s}, \mathrm{Phe}-\mathrm{CONH}), 179.5\left(\mathrm{~s}, \mathrm{PhCH}_{2} \mathrm{O}-\right.$ $\mathrm{CO}$ ), 180.1 (s, $\mathrm{SO}_{2} \mathrm{NHNHC}(=\mathrm{NH})$ ). Found: $\mathrm{C}, 56.71 ; \mathrm{H}, 4.97 ; \mathrm{N}, 16.33$. $\mathrm{C}_{24} \mathrm{H}_{26} \mathrm{~N}_{6} \mathrm{O}_{5} \mathrm{~S}$ requires: C, 56.46; $\mathrm{H}, 5.13 ; \mathrm{N}, 16.46 \%$.

4-(4-Toluenesulfonylureido-D-phenylalanylamido)-benzenesulfonyl-aminoguanidine, A34 Its m.p. $275-7^{\circ} \mathrm{C}$. ${ }^{1} \mathrm{H}-\mathrm{NMR}$ (DMSO-D $2 \mathrm{O}$ ), $\delta$, ppm: 2.67 (s, $\left.3 \mathrm{H}, \mathrm{CH}_{3} \mathrm{C}_{6} \mathrm{H}_{4}\right), 3.10-3.56\left(\mathrm{~m}, 2 \mathrm{H}, \mathrm{CH}_{2} \mathrm{CH}\right.$ of Phe), $4.11\left(\mathrm{dd},{ }^{3} J_{\mathrm{HH}}=5.0\right.$, ${ }^{3} J_{\mathrm{HH}}=7.8 ; 1 \mathrm{H}, \mathrm{CH}_{2} \mathrm{CH}$ of $\left.\mathrm{Phe}\right), 7.29-7.58\left(\mathrm{~m}, 7 \mathrm{H}, H_{\text {ortho }}\right.$ of tosyl and

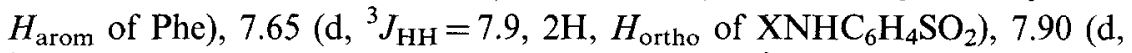
${ }^{3} J_{\mathrm{HH}}=7.9,2 \mathrm{H}, H_{\text {meta }}$ of $\left.\mathrm{XNHC}_{6} \mathrm{H}_{4} \mathrm{SO}_{2}\right), 7.95\left(\mathrm{~d},{ }^{3} J_{\mathrm{HH}}=8.3,2 \mathrm{H}, H_{\text {meta }}\right.$ of tosyl), 9.32 (br s, $\left.5 \mathrm{H}, \mathrm{SO}_{2} \mathrm{~N} H \mathrm{NHC}(=\mathrm{N} H) \mathrm{NH}_{2}\right) ;{ }^{13} \mathrm{C}-\mathrm{NMR}\left(\mathrm{DMSO}-\mathrm{D}_{2} \mathrm{O}\right.$ ), $\delta$, ppm: 26.7 (s, $\left.\mathrm{CH}_{3} \mathrm{C}_{6} \mathrm{H}_{4}\right), 41.6$ (s, $\mathrm{CH}_{2} \mathrm{CH}$ of Phe), 59.5 (s, $\mathrm{CHCH}_{2}$ of Phe), 130.8 (s, $C_{\text {para }}$ of Phe), 132.3 (s, $C_{\text {meta }}$ of $\mathrm{CH}_{3} \mathrm{C}_{6} \mathrm{H}_{4}$ ), 133.5 (s, $C_{\text {meta }}$ of $\mathrm{XNHC}_{6} \mathrm{H}_{4} \mathrm{SO}_{2}$ ), 133.9 (s, $C_{\text {meta }}$ of Phe), 134.5 (s, $C_{\text {ortho }}$ of Phe), 135.1 (s, $C_{\text {ortho }}$ of $\left.\mathrm{CH}_{3} \mathrm{C}_{6} \mathrm{H}_{4}\right), 135.5$ (s, $C_{\text {ortho }}$ of $\left.\mathrm{XNHC}_{6} \mathrm{H}_{4} \mathrm{SO}_{2}\right), 141.5$ (s, $C_{\text {ipso }}$ of Phe), 142.9 (s, $C_{\text {para }}$ of $\mathrm{XNHC}_{6} \mathrm{H}_{4} \mathrm{SO}_{2}$ ), 145.0 (s, $C_{\text {para }}$ of $\mathrm{CH}_{3} \mathrm{C}_{6} \mathrm{H}_{4}$ ), 148.4 (s, $C_{\text {ipso }}$ of $\mathrm{CH}_{3} \mathrm{C}_{6} \mathrm{H}_{4}$ ), 148.9 (s, $C_{\text {ipso }}$ of $\mathrm{XNHC}_{6} \mathrm{H}_{4} \mathrm{SO}_{2}$ ), 149.1 (s, NHCONH), 172.3 (s, Phe-CONH), 180.1 (s, $\mathrm{SO}_{2} \mathrm{NHNHC}(=\mathrm{NH})$ ). Found: $\mathrm{C}, 52.61 ; \mathrm{H}$, $5.11 ; \mathrm{N}, 14.50 . \mathrm{C}_{25} \mathrm{H}_{29} \mathrm{~N}_{6} \mathrm{O}_{6} \mathrm{~S}_{2}$ requires: $\mathrm{C}, 52.44 ; \mathrm{H}, 4.93 ; \mathrm{N}, 14.68 \%$.

4-(4-Toluenesulfonylureido-L-prolylamido)-benzenesulfonyl-aminoguanidine, A35 Its m.p. $275-7^{\circ} \mathrm{C}$ : ${ }^{1} \mathrm{H}-\mathrm{NMR}$ (DMSO-D $2 \mathrm{O}$ ), $\delta$, ppm: 1.18-1.38 (m, 1H, HCH of Pro), 1.55-1.65 (m, 1H, HCH), $1.70-1.85\left(\mathrm{~m}, 2 \mathrm{H}, \mathrm{CH}_{2}\right.$ of Pro), 2.64 (s, $3 \mathrm{H}, \mathrm{CH}_{3} \mathrm{C}_{6} \mathrm{H}_{4}$ ), 3.16-3.30 (m, 2H, $\mathrm{CH}_{2} \mathrm{~N}$ of Pro), 3.75-3.80 (m, $1 \mathrm{H}, \mathrm{CHCO}$ of Pro), 7.42 (d, ${ }^{3} J_{\mathrm{HH}}=8.3,2 \mathrm{H}, H_{\text {ortho }}$ of tosyl), 7.65 (d, ${ }^{3} J_{\mathrm{HH}}=7.9,2 \mathrm{H}, H_{\text {ortho }}$ of $\left.\mathrm{XNHC}_{6} \mathrm{H}_{4} \mathrm{SO}_{2}\right), 7.90\left(\mathrm{~d},{ }^{3} J_{\mathrm{HH}}=7.9,2 \mathrm{H}, H_{\text {meta }}\right.$ of $\mathrm{XNHC}_{6} \mathrm{H}_{4} \mathrm{SO}_{2}$ ), $7.95\left(\mathrm{~d},{ }^{3} J_{\mathrm{HH}}=8.3,2 \mathrm{H}, H_{\text {meta }}\right.$ of tosyl), 9.32 (br s, $5 \mathrm{H}$, $\left.\mathrm{SO}_{2} \mathrm{NHNHC}(=\mathrm{NH}) \mathrm{NH}_{2}\right) ;{ }^{13} \mathrm{C}-\mathrm{NMR}\left(\mathrm{DMSO}-\mathrm{D}_{2} \mathrm{O}\right), \delta, \mathrm{ppm}: 15.6\left(\mathrm{~s}, \mathrm{CH}_{2}\right.$ of Pro), 21.3 (s, $\mathrm{CH}_{2}$ of Pro), 26.7 (s, $\mathrm{CH}_{3} \mathrm{C}_{6} \mathrm{H}_{4}$ ), 46.9 (s, $\mathrm{CH}_{2} \mathrm{~N}$ of Pro), 64.5 (s, $C$ HCO of Pro), 132.4 (s, $C_{\text {meta }}$ of $\mathrm{CH}_{3} \mathrm{C}_{6} \mathrm{H}_{4}$ ), 133.5 (s, $C_{\text {meta }}$ of XNH$\mathrm{C}_{6} \mathrm{H}_{4} \mathrm{SO}_{2}$ ), 135.1 (s, $C_{\text {ortho }}$ of $\mathrm{CH}_{3} \mathrm{C}_{6} \mathrm{H}_{4}$ ), 135.5 (s, $C_{\text {ortho }}$ of $\mathrm{XNHC}_{6} \mathrm{H}_{4} \mathrm{SO}_{2}$ ), $142.9\left(\mathrm{~s}, C_{\text {para }}\right.$ of $\left.\mathrm{XNHC}_{6} \mathrm{H}_{4} \mathrm{SO}_{2}\right), 145.0\left(\mathrm{~s}, C_{\text {para }}\right.$ of $\left.\mathrm{CH}_{3} \mathrm{C}_{6} \mathrm{H}_{4}\right), 148.4(\mathrm{~s}$, $C_{\text {ipso }}$ of $\left.\mathrm{CH}_{3} \mathrm{C}_{6} \mathrm{H}_{4}\right), 148.9$ (s, $C_{\text {ipso }}$ of $\mathrm{XNHC}_{6} \mathrm{H}_{4} \mathrm{SO}_{2}$ ), 149.1 (s, NHCONH), 175.6 (s, Pro- $C \mathrm{ONH}), 180.1$ (s, $\mathrm{SO}_{2} \mathrm{NHNHC}(=\mathrm{NH})$ ). Found: $\mathrm{C}, 45.69 ; \mathrm{H}$, $5.00 ; \mathrm{N}, 18.63 . \mathrm{C}_{20} \mathrm{H}_{25} \mathrm{~N}_{7} \mathrm{O}_{6} \mathrm{~S}_{2}$ requires: $\mathrm{C}, 45.88 ; \mathrm{H}, 4.81 ; \mathrm{N}, 18.73 \%$.

4-(4-Toluenesulfonylureido-D-phenylalanyl-L-prolylamido)-benzenesulfonylaminoguanidine, $\mathbf{A} 36$ Its m.p. $281-3^{\circ} \mathrm{C}$ (dec.). ${ }^{1} \mathrm{H}-\mathrm{NMR}\left(\mathrm{DMSO}-\mathrm{D}_{2} \mathrm{O}\right.$ ), $\delta$, ppm: $1.19-1.40(\mathrm{~m}, 1 \mathrm{H}, H \mathrm{CH}$ of Pro), $1.55-1.69(\mathrm{~m}, 1 \mathrm{H}, \mathrm{HCH}), 1.75-1.85$ 
(m, $2 \mathrm{H}, \mathrm{CH}_{2}$ of Pro). 2.66 (s. $\left.3 \mathrm{H}, \mathrm{CH}_{3} \mathrm{C}_{6} \mathrm{H}_{4}\right), 3.10-3.56\left(\mathrm{~m}, 2 \mathrm{H}, \mathrm{CH}_{2} \mathrm{CH}\right.$ of Phe), $3.20-3.35\left(\mathrm{~m}, 2 \mathrm{H}, \mathrm{CH}_{2} \mathrm{~N}\right.$ of Pro). 3.75-3.80 (m, $1 \mathrm{H}, \mathrm{CHCO}$ of Pro), $4.12\left(\right.$ dd. ${ }^{3} J_{\mathrm{HH}}=5.0,{ }^{3} J_{\mathrm{HH}}=7.8 ; 1 \mathrm{H}, \mathrm{CH}_{2} \mathrm{CH}$ of Phe), $7.29-7.58(\mathrm{~m}, 7 \mathrm{H}$, $H_{\text {ortho }}$ of tosyl and $H_{\text {arom }}$ of Phe). 7.65 (d. ${ }^{3} J_{\mathrm{HH}}=7.9,2 \mathrm{H}, H_{\text {ortho }}$ of $\left.\mathrm{XNHC}_{6} \mathrm{H}_{4} \mathrm{SO}_{2}\right), 7.90\left(\mathrm{~d},{ }^{3} J_{\mathrm{HH}}=7.9,2 \mathrm{H}, H_{\text {meta }}\right.$ of $\left.\mathrm{XNHC}_{6} \mathrm{H}_{4} \mathrm{SO}_{2}\right), 7.95(\mathrm{~d}$, ${ }^{3} J_{\mathrm{HH}}=8.3,2 \mathrm{H}, H_{\text {meta }}$ of tosyl), $9.32\left(\right.$ br s, $\left.5 \mathrm{H}, \mathrm{SO}_{2} \mathrm{~N} H \mathrm{NHC}(=\mathrm{N} H) \mathrm{N} H_{2}\right)$; ${ }^{13} \mathrm{C}-\mathrm{NMR}$ (DMSO-D $2 \mathrm{O}$ ), $\delta, \mathrm{ppm}$ : 15.5 (s, $\mathrm{CH}_{2}$ of Pro), 21.5 (s, $\mathrm{CH}_{2}$ of Pro), 26.9 (s, $\mathrm{CH}_{3} \mathrm{C}_{6} \mathrm{H}_{4}$ ), 41.6 (s, $\mathrm{CH}_{2} \mathrm{CH}$ of Phe), 46.9 (s, $\mathrm{CH}_{2} \mathrm{~N}$ of Pro), 59.5 (s. $\mathrm{CHCH}_{2}$ of Phe), 64.5 (s, $\mathrm{CHCO}$ of Pro), 130.2 (s, $C_{\text {para }}$ of Phe), 132.5 (s, $C_{\text {meta }}$ of $\mathrm{CH}_{3} \mathrm{C}_{6} \mathrm{H}_{4}$ ), 133.5 (s, $\mathrm{C}_{\text {meta }}$ of $\mathrm{XNHC}_{6} \mathrm{H}_{4} \mathrm{SO}_{2}$ ), 133.9 (s, $C_{\text {meta }}$ of Phe), 134.7 (s, $C_{\text {ortho }}$ of Phe), $135.0\left(\mathrm{~s}, C_{\text {ortho }}\right.$ of $\mathrm{CH}_{3} \mathrm{C}_{6} \mathrm{H}_{4}$ ), 135.5 (s, $C_{\text {ortho }}$ of $\mathrm{XNHC}_{6} \mathrm{H}_{4} \mathrm{SO}_{2}$ ), 141.5 (s, $C_{\text {ipso }}$ of Phe), 142.6 (s, $C_{\text {para }}$ of XNH$\left.\mathrm{C}_{6} \mathrm{H}_{4} \mathrm{SO}_{2}\right), 145.3\left(\mathrm{~s}, \mathrm{C}_{\text {para }}\right.$ of $\left.\mathrm{CH}_{3} \mathrm{C}_{6} \mathrm{H}_{4}\right), 148.4\left(\mathrm{~s}, \mathrm{C}_{\text {ipso }}\right.$ of $\left.\mathrm{CH}_{3} \mathrm{C}_{6} \mathrm{H}_{4}\right), 148.9$ (s. $C_{\text {ipso }}$ of $\mathrm{XNHC}_{6} \mathrm{H}_{4} \mathrm{SO}_{2}$ ), 149.1 (s, NHCONH), 172.3 (s, Phe-CONH), 174.0 (Pro- $\mathrm{CONH}$ ), 180.1 (s, $\mathrm{SO}_{2} \mathrm{NHNHC}(=\mathrm{NH})$ ). Found: $\mathrm{C}, 52.07 ; \mathrm{H}$, 5.18; N, 16.54. $\mathrm{C}_{29} \mathrm{H}_{34} \mathrm{~N}_{8} \mathrm{O}_{7} \mathrm{~S}_{2}$ requires: C. 51.93; H, 5.11; N, 16.71\%.

4-( $\mathrm{N}$-Benzyloxycarbony-D-phenylalanylprolylamido)-benzenesulfonyl-aminoguanidine, A37 Its m.p. $258-61^{\circ} \mathrm{C} .{ }^{1} \mathrm{H}-\mathrm{NMR}\left(\mathrm{DMSO}_{2} \mathrm{O}\right), \delta, \mathrm{ppm}$ : $1.19-1.40(\mathrm{~m}, 1 \mathrm{H}, \mathrm{HCH}$ of Pro), $1.55-1.69(\mathrm{~m}, 1 \mathrm{H}, \mathrm{HCH}), 1.75-1.85(\mathrm{~m}$, $2 \mathrm{H}, \mathrm{CH}_{2}$ of Pro), 3.10-3.56 (m, 2H, CH $\mathrm{H}_{2} \mathrm{CH}$ of Phe), 3.20-3.35 (m, $2 \mathrm{H}$, $\mathrm{CH}_{2} \mathrm{~N}$ of Pro), 3.75-3.80 (m, $1 \mathrm{H}, \mathrm{CHCO}$ of Pro), $4.11\left(\mathrm{dd},{ }^{3} J_{\mathrm{HH}}=5.0\right.$, ${ }^{3} J_{\mathrm{HH}}=7.8 ; 1 \mathrm{H}, \mathrm{CH}_{2} \mathrm{CH}$ of Phe), $5.10\left(\mathrm{~s}, 2 \mathrm{H}, \mathrm{PhCH} \mathrm{H}_{2} \mathrm{O}\right), 7.20-7.42(\mathrm{~m}, 10 \mathrm{H}$, $H_{\text {arom }}$ of Phe and $\left.\mathrm{PhCH}_{2} \mathrm{O}\right), 7.65$ (d. ${ }^{3} J_{\mathrm{HH}}=7.9,2 \mathrm{H}, H_{\text {ortho }}$ of XNH$\left.\mathrm{C}_{6} \mathrm{H}_{4} \mathrm{SO}_{2}\right), 7.90\left(\mathrm{~d},{ }^{3} J_{\mathrm{HH}}=7.9,2 \mathrm{H}, \mathrm{H}_{\text {meta }}\right.$ of $\left.\mathrm{XNHC}_{6} \mathrm{H}_{4} \mathrm{SO}_{2}\right), 9.32$ (br s, $5 \mathrm{H}$, $\left.\mathrm{SO}_{2} \mathrm{~N} H \mathrm{NHC}(=\mathrm{N} H) \mathrm{NH}_{2}\right) ;{ }^{13} \mathrm{C}-\mathrm{NMR}\left(\mathrm{DMSO}-\mathrm{D}_{2} \mathrm{O}\right), \delta, \mathrm{ppm}: 15.0\left(\mathrm{~s}, \mathrm{CH}_{2}\right.$ of Pro), 21.9 (s, $\mathrm{CH}_{2}$ of Pro), 41.8 (s. $\mathrm{CH}_{2} \mathrm{CH}$ of Phe), 46.7 (s, $\mathrm{CH}_{2} \mathrm{~N}$ of Pro), 59.9 (s, $\mathrm{CHCH}_{2}$ of Phe), 64.1 (s, $\mathrm{CHCO}$ of Pro), 75.0 (s, $\mathrm{PhCH}_{2} \mathrm{O}$ ), 130.8 (s, $C_{\text {para }}$ of Phe). 133.6 (s, $C_{\text {meta }}$ of $\mathrm{XNHC}_{6} \mathrm{H}_{4} \mathrm{SO}_{2}$ ), 133.4 (s, $C_{\text {meta }}$ of Phe), 134.3 (s, $C_{\text {ortho }}$ of Phe), 135.7 (s, $C_{\text {ortho }}$ of $\mathrm{XNHC}_{6} \mathrm{H}_{4} \mathrm{SO}_{2}$ ), 141.2 (s, $C_{\text {ipso }}$ of Phe), $142.8\left(\mathrm{~s}, C_{\text {para }}\right.$ of $\left.\mathrm{XNHC}_{6} \mathrm{H}_{4} \mathrm{SO}_{2}\right), 148.9\left(\mathrm{~s}, \mathrm{C}_{\text {ipso }}\right.$ of $\mathrm{XNHC}_{6} \mathrm{H}_{4}{ }^{-}$ $\mathrm{SO}_{2}$ ), 173.3 (s, Phe-CONH), 174.3 (Pro-CONH), 179.5 (s, $\mathrm{PhCH}_{2} \mathrm{OCO}$ ), 180.4 (s, $\mathrm{SO}_{2} \mathrm{NHNHC}(=\mathrm{NH})$ ). Found: C. $57.40: \mathrm{H}, 5.58 ; \mathrm{N}, 16.09 . \mathrm{C}_{29} \mathrm{H}_{33^{-}}$ $\mathrm{N}_{7} \mathrm{O}_{6} \mathrm{~S}$ requires: C. 57.32; $\mathrm{H}, 5.47: \mathrm{N}, 16.13 \%$.

4-(4-Toluenesulfonylureido-glycyl-L-histidinylamido)-benzenesulfonyl-aminoguanidine, $\mathbf{A 3 8}$ Its m.p. $282-3^{\circ} \mathrm{C}$. ${ }^{1} \mathrm{H}-\mathrm{NMR}$ (DMSO-D $2 \mathrm{O}$ ), $\delta, \mathrm{ppm:} 2.64$ (s. $3 \mathrm{H}, \mathrm{CH}_{3} \mathrm{C}_{6} \mathrm{H}_{4}$ ), 3.67 (s, 2H, $\mathrm{CH}_{2}$ of Gly), 3.35-3.46 (m, $2 \mathrm{H}, \mathrm{CHCH}_{2}$ of His), 4.59-4.67 (m, 1H, $\mathrm{CHCH}_{2}$ of His), 7.34 (s, $1 \mathrm{H}, \mathrm{CH}-5$ of His), 7.53 $\left(\mathrm{d},{ }^{3} J_{\mathrm{HH}}=8.1,2 \mathrm{H}, H_{\text {ortho }}\right.$ of $\left.\mathrm{CH}_{3} \mathrm{C}_{6} \mathrm{H}_{4}\right) .7 .68\left(\mathrm{~d},{ }^{3} J_{\mathrm{HH}}=7.9,2 \mathrm{H}, H_{\text {ortho }}\right.$ of $\left.\mathrm{XNHC}_{6} \mathrm{H}_{4} \mathrm{SO}_{2}\right), 7.87$ (d, ${ }^{3} J_{\mathrm{HH}}=8.1,2 \mathrm{H}, \quad H_{\text {meta }}$ of $\left.\mathrm{CH}_{3} \mathrm{C}_{6} \mathrm{H}_{4}\right), 7.95$ (d. ${ }^{3} J_{\mathrm{HH}}=7.9,2 \mathrm{H}, H_{\text {meta }}$ of $\mathrm{XNHC}_{6} \mathrm{H}_{4} \mathrm{SO}_{2}$ ), 8.35 (s, $1 \mathrm{H}, \mathrm{CH}-2$ of His), 9.29 
(br s, $\left.5 \mathrm{H}, \mathrm{SO}_{2} \mathrm{NHNHC}(=\mathrm{NH}) \mathrm{NH}_{2}\right) ;{ }^{13} \mathrm{C}-\mathrm{NMR}\left(\mathrm{DMSO}-\mathrm{D}_{2} \mathrm{O}\right), \delta$, ppm: 26.1 (s, $\mathrm{CH}_{3} \mathrm{C}_{6} \mathrm{H}_{4}$ ), 40.5 (s, $\mathrm{CH}_{2}$ of Gly), 59.6 (s, $\mathrm{CHCH}_{2}$ of His), 122.2 (s, $C-4$ of His), 130.7 (s, $C_{\text {meta }}$ of $\left.\mathrm{CH}_{3} \mathrm{C}_{6} \mathrm{H}_{4}\right), 130.9$ (s, $C_{\text {meta }}$ of $\left.\mathrm{XNHC}_{6} \mathrm{H}_{4} \mathrm{SO}_{2}\right), 131.8$ (s, C-5 of His), 134.2 (s, $C_{\text {ortho }}$ of $\mathrm{CH}_{3} \mathrm{C}_{6} \mathrm{H}_{4}$ ), 135.0 (s, $C_{\text {ortho }}$ of XNH$\mathrm{C}_{6} \mathrm{H}_{4} \mathrm{SO}_{2}$ ), 137.2 (s, $C-2$ of His), 139.1 (s, $C_{\text {para }}$ of $\mathrm{CH}_{3} \mathrm{C}_{6} \mathrm{H}_{4}$ ), 140.4 (s, $C_{\text {para }}$ of $\left.\mathrm{XNHC}_{6} \mathrm{H}_{4} \mathrm{SO}_{2}\right), 145.6\left(\mathrm{~s}, C_{\text {ipso }}\right.$ of $\left.\mathrm{CH}_{3} \mathrm{C}_{6} \mathrm{H}_{4}\right), 148.5\left(\mathrm{~s}, C_{\text {ipso }}\right.$ of XNH$\mathrm{C}_{6} \mathrm{H}_{4} \mathrm{SO}_{2}$ ), 149.1 (s, NHCONH), 175.0 (s, $\mathrm{CH}_{2} \mathrm{CO}$ of Gly), 176.4 (s, CONH of His), 183.2 (s, $\mathrm{SO}_{2} \mathrm{NHC}(=\mathrm{NH})$ ). Found: $\mathrm{C}, 44.64 ; \mathrm{H}, 4.39 ; \mathrm{N}, 22.51$. $\mathrm{C}_{23} \mathrm{H}_{28} \mathrm{~N}_{10} \mathrm{O}_{7} \mathrm{~S}_{2}$ requires: $\mathrm{C}, 44.51 ; \mathrm{H}, 4.55 ; \mathrm{N}, 22.57 \%$.

4-(4-Toluenesulfonylureido- $\beta$-alanyl-L-histidinylamido)-benzenesulfonylaminoguanidine, A39 Its m.p. $290-1^{\circ} \mathrm{C} .{ }^{1} \mathrm{H}-\mathrm{NMR}\left(\mathrm{DMSO}-\mathrm{D}_{2} \mathrm{O}\right), \delta$, ppm: $2.64\left(\mathrm{~s}, 3 \mathrm{H}, \mathrm{CH}_{3} \mathrm{C}_{6} \mathrm{H}_{4}\right), 2.79-2.89\left(\mathrm{~m}, 2 \mathrm{H}, \mathrm{CH}_{2}\right.$ of $\beta$-Ala), $3.11-3.27(\mathrm{~m}, 2 \mathrm{H}$, $\mathrm{CH}_{2}$ of $\beta$-Ala), 3.34-3.44 (m, $2 \mathrm{H}, \mathrm{CHCH}_{2}$ of His), 4.57-4.65 (m, $1 \mathrm{H}$, $\mathrm{CHCH}_{2}$ of His), 7.31 (s, $1 \mathrm{H}, \mathrm{CH}-5$ of His), $7.56\left(\mathrm{~d},{ }^{3} J_{\mathrm{HH}}=8.1,2 \mathrm{H}, H_{\text {ortho }}\right.$ of $\left.\mathrm{CH}_{3} \mathrm{C}_{6} \mathrm{H}_{4}\right), 7.69\left(\mathrm{~d},{ }^{3} J_{\mathrm{HH}}=7.9,2 \mathrm{H}, \quad H_{\text {ortho }}\right.$ of $\left.\mathrm{XNHC}_{6} \mathrm{H}_{4} \mathrm{SO}_{2}\right), 7.88$ (d, ${ }^{3} J_{\mathrm{HH}}=8.1,2 \mathrm{H}, H_{\text {meta }}$ of $\left.\mathrm{CH}_{3} \mathrm{C}_{6} \mathrm{H}_{4}\right), 7.94\left(\mathrm{~d},{ }^{3} J_{\mathrm{HH}}=7.9,2 \mathrm{H}, H_{\text {meta }}\right.$ of $\mathrm{XNHC}_{6} \mathrm{H}_{4} \mathrm{SO}_{2}$ ), 8.35 (s, $1 \mathrm{H}, \mathrm{CH}-2$ of $\mathrm{His}$ ), 9.29 (br s, $5 \mathrm{H}, \mathrm{SO}_{2} \mathrm{NHNHC}-$ $\left.(=\mathrm{NH}) \mathrm{NH}_{2}\right) ;{ }^{13} \mathrm{C}-\mathrm{NMR}\left(\mathrm{DMSO}-\mathrm{D}_{2} \mathrm{O}\right), \delta$, ppm: $25.8\left(\mathrm{~s}, \mathrm{CH}_{3} \mathrm{C}_{6} \mathrm{H}_{4}\right), 33.4(\mathrm{~s}$, $\mathrm{CH}_{2}$ of His), 37.5 (s, $\mathrm{NHCH}_{2} \mathrm{CH}_{2}$ of $\beta$-Ala), 40.8 (s, $\mathrm{CH}_{2} \mathrm{CH}_{2} \mathrm{CO}$ of $\beta$-Ala), 59.6 (s, $\mathrm{CHCH}_{2}$ of His), 122.2 (s, $C-4$ of His), 130.6 (s, $C_{\text {meta }}$ of $\mathrm{CH}_{3} \mathrm{C}_{6} \mathrm{H}_{4}$ ), 130.9 (s, $C_{\text {meta }}$ of $\mathrm{XNHC}_{6} \mathrm{H}_{4} \mathrm{SO}_{2}$ ), 131.8 (s, C-5 of His), 134.2 (s, $C_{\text {ortho }}$ of $\mathrm{CH}_{3} \mathrm{C}_{6} \mathrm{H}_{4}$ ), 135.1 (s, $C_{\text {ortho }}$ of $\mathrm{XNHC}_{6} \mathrm{H}_{4} \mathrm{SO}_{2}$ ), 137.2 (s, C-2 of His), 139.1 (s, $C_{\text {para }}$ of $\left.\mathrm{CH}_{3} \mathrm{C}_{6} \mathrm{H}_{4}\right), 140.4$ (s, $C_{\text {para }}$ of $\mathrm{XNHC}_{6} \mathrm{H}_{4} \mathrm{SO}_{2}$ ), 145.6 (s, $C_{\text {ipso }}$ of $\mathrm{CH}_{3} \mathrm{C}_{6} \mathrm{H}_{4}$ ), 148.5 (s, $C_{\text {ipso }}$ of $\mathrm{XNHC}_{6} \mathrm{H}_{4} \mathrm{SO}_{2}$ ), 149.1 (s, NHCONH), 175.6 (s, $\mathrm{CH}_{2} \mathrm{CO}$ of $\beta$-Ala), 176.4 (s, CONH of His), 183.2 (s, $\mathrm{SO}_{2} \mathrm{~N}=C$ ). Found: $\mathrm{C}$, 45.56; $\mathrm{H}, 4.75 ; \mathrm{N}, 21.95 . \mathrm{C}_{24} \mathrm{H}_{30} \mathrm{~N}_{10} \mathrm{O}_{7} \mathrm{~S}_{2}$ requires: $\mathrm{C}, 45.42 ; \mathrm{H}, 4.76 ; \mathrm{N}, 22.07 \%$.

4-(4-Toluenesulfonylureido-L-prolyl-glycylamido)-benzenesulfonyl-aminoguanidine, A40 Its m.p. $239-40^{\circ} \mathrm{C} .{ }^{1} \mathrm{H}-\mathrm{NMR}$ (DMSO- $\mathrm{D}_{2} \mathrm{O}$ ), $\delta$, ppm: $1.18-$ $1.38(\mathrm{~m}, 1 \mathrm{H}, H \mathrm{CH}$ of Pro), 1.55-1.65 (m, 1H, HCH), 1.70-1.85 (m, 2H, $\mathrm{CH}_{2}$ of Pro), $2.63\left(\mathrm{~s}, 3 \mathrm{H}, \mathrm{CH}_{3} \mathrm{C}_{6} \mathrm{H}_{4}\right), 3.16-3.30\left(\mathrm{~m}, 2 \mathrm{H}, \mathrm{CH}_{2} \mathrm{~N}\right.$ of Pro), 3.67 (s, $2 \mathrm{H}, \mathrm{CH}_{2}$ of Gly), 3.75-3.80 (m, $1 \mathrm{H}, \mathrm{CHCO}$ of Pro), $7.65\left(\mathrm{~d},{ }^{3} \mathrm{~J}_{\mathrm{HH}}=8.1\right.$, $2 \mathrm{H}, H_{\text {ortho }}$ of tosyl), $7.69\left(\mathrm{~d},{ }^{3} \mathrm{~J}_{\mathrm{HH}}=7.9,2 \mathrm{H}, H_{\text {ortho }}\right.$ of $\left.\mathrm{XNHC}_{6} \mathrm{H}_{4} \mathrm{SO}_{2}\right), 7.90$ (d, ${ }^{3} J_{\mathrm{HH}}=7.9,2 \mathrm{H}, H_{\text {meta }}$ of $\mathrm{XNHC}_{6} \mathrm{H}_{4} \mathrm{SO}_{2}$ ), 7.99 (d, ${ }^{3} J_{\mathrm{HH}}=8.1,2 \mathrm{H}$, $H_{\text {meta }}$ of tosyl), $9.36\left(\mathrm{br} \mathrm{s}, 5 \mathrm{H}, \mathrm{SO}_{2} \mathrm{~N} H \mathrm{~N} H \mathrm{C}(=\mathrm{N} H) \mathrm{NH}_{2}\right) ;{ }^{13} \mathrm{C}-\mathrm{NMR}$ (DMSO- $\mathrm{D}_{2} \mathrm{O}$ ), $\delta$, ppm: 15.6 (s, $\mathrm{CH}_{2}$ of Pro), 21.3 (s, $\mathrm{CH}_{2}$ of Pro), 26.1 (s, $\mathrm{CH}_{3} \mathrm{C}_{6} \mathrm{H}_{4}$ ), 40.8 (s, $\mathrm{CH}_{2}$ of Gly), 46.4 (s, $\mathrm{CH}_{2} \mathrm{~N}$ of Pro), 64.7 (s, $\mathrm{CHCO}$ of Pro), 132.4 (s, $C_{\text {meta }}$ of $\mathrm{CH}_{3} \mathrm{C}_{6} \mathrm{H}_{4}$ ), 133.5 (s, $C_{\text {meta }}$ of $\mathrm{XNHC}_{6} \mathrm{H}_{4} \mathrm{SO}_{2}$ ), 135.0 (s, $C_{\text {ortho }}$ of $\mathrm{CH}_{3} \mathrm{C}_{6} \mathrm{H}_{4}$ ), 135.5 (s, $C_{\text {ortho }}$ of $\mathrm{XNHC}_{6} \mathrm{H}_{4} \mathrm{SO}_{2}$ ), 142.9 (s, $C_{\text {para }}$ of $\mathrm{XNHC}_{6} \mathrm{H}_{4} \mathrm{SO}_{2}$ ), 145.0 (s, $C_{\text {para }}$ of $\left.\mathrm{CH}_{3} \mathrm{C}_{6} \mathrm{H}_{4}\right), 148.4$ (s, $C_{\text {ipso }}$ of $\mathrm{CH}_{3}$ $\mathrm{C}_{6} \mathrm{H}_{4}$ ), 148.9 (s, $C_{\text {ipso }}$ of $\mathrm{XNHC}_{6} \mathrm{H}_{4} \mathrm{SO}_{2}$ ), 149.1 (s, NHCONH), 175.6 
(s, Pro-CONH). 176.3 (s, CONH of Gly), 180.1 (s, $\mathrm{SO}_{2} \mathrm{NHNHC}(=\mathrm{NH})$ ), Found: $\mathrm{C}, 45.38: \mathrm{H}, 4.77: \mathrm{N}, 19.21 . \mathrm{C}_{22} \mathrm{H}_{28} \mathrm{~N}_{8} \mathrm{O}_{7} \mathrm{~S}_{2}$ requires: $\mathrm{C}, 45.51 ; \mathrm{H}$, $4.86 ; \mathrm{N}, 19.30 \%$.

l-N-[(4-Guanidinoaminosulfony/-phenyl) aminocarbonylpropyl]-2,4,6-trimethylpyridinium perchlorate, B1 As white crystals, m.p. $254-5^{\circ} \mathrm{C}$ (yield of $71 \%)$. IR (KBr), $\mathrm{cm}^{-1}: 625,712,1100,1170,1341,1540,1580,1645,1675$, 3060, 3250, 3335: ${ }^{1} \mathrm{H}-\mathrm{NMR}$ (TFA), $\delta, \mathrm{ppm}: 1.90\left(\mathrm{~m}, 2 \mathrm{H}, 3-\mathrm{CH}_{2}\right.$ of GABA moiety), 2.28 (t, $2 \mathrm{H}, \alpha-\mathrm{CH}_{2}$ of GABA moiety), 2.65 (s, 3H, 4-Me), 2.90 (s, $\left.6 \mathrm{H}, 2,6-(\mathrm{Me})_{2}\right), 3.01\left(\mathrm{t}, 2 \mathrm{H}, \gamma-\mathrm{CH}_{2}\right.$ of GABA moiety), 7.47-8.38 (m, 6H, ArH from 1,4-phenylene and 3,5-H from pyridinium). Found: $\mathrm{C}, 43.74 ; \mathrm{H}$, $5.09 ; \mathrm{N}, 16.03 . \mathrm{C}_{19} \mathrm{H}_{27} \mathrm{~N}_{6} \mathrm{O}_{3} \mathrm{~S}^{-} \mathrm{ClO}_{4}^{-}$requires: $\mathrm{C}, 43.57 ; \mathrm{H}, 5.24 ; \mathrm{N}, 16.19 \%$.

l-N-[(4-Guanidinoaminosulfony/-phenl) aminocarbonylpropyl]-2-iso-prop.7-4,6-dimethylpyidinium perchlorate, B2 As white crystals, m.p. 240 $1{ }^{\circ} \mathrm{C}$ (yield of $65 \%$ ). IR ( $\mathrm{KBr}$ ), $\mathrm{cm}^{-1}: 625,685,1100,1175,1285,1345$, $1540,1645,1675,3040,3255,3380 ;{ }^{1} \mathrm{H}-\mathrm{NMR}$ (TFA). $\delta, \mathrm{ppm}: 1.47(\mathrm{~d}, 6 \mathrm{H}$, 2Me from $i-\mathrm{Pr}), 1.90\left(\mathrm{~m}, 2 \mathrm{H}, 3-\mathrm{CH}_{2}\right.$ of $\mathrm{GABA}$ moiety), $2.28\left(\mathrm{t}, 2 \mathrm{H}, \alpha-\mathrm{CH}_{2}\right.$ of GABA moiety), $2.68(\mathrm{~s}, 3 \mathrm{H}, 4-\mathrm{Me}), 2.90(\mathrm{~s}, 3 \mathrm{H}, 6-\mathrm{Me}), 3.01\left(\mathrm{t}, 2 \mathrm{H}, \gamma-\mathrm{CH}_{2}\right.$ of GABA moiety), 3.10-3.55 (m, $1 \mathrm{H}, \mathrm{CH}$ from $i$-Pr), $7.30-8.49(\mathrm{~m}, 6 \mathrm{H}$, ArH from 1,4-phenylene and 3,5-H from pyridinium). Found: $\mathrm{C}, 45.97 ; \mathrm{H}$, 5.76; N, 15.30. $\mathrm{C}_{21} \mathrm{H}_{31} \mathrm{~N}_{6} \mathrm{O}_{3} \mathrm{~S}^{+} \mathrm{ClO}_{4}^{-}$requires: $\mathrm{C}, 46.11 ; \mathrm{H}, 5.71 ; \mathrm{N}, 15.36 \%$.

l-N-[ (4-Guanidinoaminosulfonyl-phenyl) aminocarbonylpropyl]-2,6-di-isopropyl-4-methlpyidinium perchlorate, B3 As white crystals, m.p. 247$9^{\circ} \mathrm{C}$ (yield of $38 \%$ ). IR $(\mathrm{KBr}), \mathrm{cm}^{-1}: 625,682,1100,1175,1285,1345,1540$, 1645, 1675, 3040, 3235, 3300; ' H-NMR (TFA), $\delta, p p m: 1.48$ (d, 12H, 4Me from $2 i$-Pr). $1.90\left(\mathrm{~m}, 2 \mathrm{H}, 3-\mathrm{CH}_{2}\right.$ of $\mathrm{GABA}$ moiety), $2.29\left(\mathrm{t}, 2 \mathrm{H}, \alpha-\mathrm{CH}_{2}\right.$ of GABA moiety). $2.70(\mathrm{~s}, 3 \mathrm{H}, 4-\mathrm{Me}), 3.01$ (t, $2 \mathrm{H}, \gamma-\mathrm{CH}_{2}$ of GABA moiety), 3.15-3.26 (m, 2H, 2CH from $2 i-\mathrm{Pr}), 7.33-8.40(\mathrm{~m}, 6 \mathrm{H}$, ArH from 1,4-phenylene and 3,5-H from pyridinium). Found: C. 47.87; H, 5.95; N, 14.54. $\mathrm{C}_{23} \mathrm{H}_{35} \mathrm{~N}_{6} \mathrm{O}_{3} \mathrm{~S}^{+} \mathrm{ClO}_{4}$ requires: C, 48.04: H. 6.13: N, $14.61 \%$.

l-N-[ 4-Guanidinoaminosulfonl-phenly / aminocarbonylpropyl]-2,6-dimethyl4-phenylpyridinium perchlorate, B4 As white crystals, m.p. $198-9^{\circ} \mathrm{C}$ (yield of $39 \%)$. IR $(\mathrm{KBr}), \mathrm{cm}^{-1}: 625,665,770,1100,1175,1285,1345,1540,1580$, $1645,1675,3050,3360 ;{ }^{1} \mathrm{H}-\mathrm{NMR}$ (TFA). $\delta, \mathrm{ppm}: 1.90\left(\mathrm{~m}, 2 \mathrm{H}, \beta-\mathrm{CH}_{2}\right.$ of GABA moiety). 2.28 (t. $2 \mathrm{H}, \alpha-\mathrm{CH}_{2}$ of GABA moiety), $3.00\left(\mathrm{t}, 2 \mathrm{H}, \gamma-\mathrm{CH}_{2}\right.$ of GABA moiety), $3.08\left(\mathrm{~s}, 6 \mathrm{H}, 2,6-(\mathrm{Me})_{2}\right), 7.50-8.41(\mathrm{~m}, 11 \mathrm{H}, \mathrm{ArH}$ from 1,4phenylene. 4-Ph and 3.5-H from pyridinium). Found: $\mathrm{C}, 49.78 ; \mathrm{H}, 5.12 ; \mathrm{N}$, 14.29. $\mathrm{C}_{24} \mathrm{H}_{29} \mathrm{~N}_{6} \mathrm{O}_{3} \mathrm{~S}^{+} \mathrm{ClO}_{4}$ requires: $\mathrm{C}, 49.61 ; \mathrm{H}, 5.03 ; \mathrm{N}, 14.46 \%$.

l-N-[ (4-Guanidinoaminosulfony/-phenll) aminocarbonlpropyl]-2,6-diethyl4-phenylpyridinium perchlorate, B5 As white crystals, m.p. $240-1{ }^{\circ} \mathrm{C}$ (yield of $43 \%)$. IR $(\mathrm{KBr}), \mathrm{cm}^{-1}: 625.700,780,1100,1173,1285,1345,1540,1580$, 
1645, 1675, 3060, 3240, 3330; ${ }^{1} \mathrm{H}-\mathrm{NMR}$ (TFA), $\delta$, ppm: 1.90 (m, $2 \mathrm{H}, \beta-\mathrm{CH}_{2}$ of GABA moiety), $2.28\left(\mathrm{t}, 2 \mathrm{H}, \alpha-\mathrm{CH}_{2}\right.$ of GABA moiety), $1.67(\mathrm{t}, 6 \mathrm{H}, 2 \mathrm{Me}$ from $\mathrm{Et}), 3.01$ (t, $2 \mathrm{H}, \gamma-\mathrm{CH}_{2}$ of GABA moiety), 3.15-3.80 (m, $4 \mathrm{H}$, $2 \mathrm{CH}_{2}$ from $\left.\mathrm{Et}\right), 7.42-8.50(\mathrm{~m}, 11 \mathrm{H}, \mathrm{ArH}$ from 1,4-phenylene, 4-Ph and 3,5-H from pyridinium). Found: $\mathrm{C}, 51.07 ; \mathrm{H}, 5.65 ; \mathrm{N}, 13.54 . \mathrm{C}_{26} \mathrm{H}_{33} \mathrm{~N}_{6} \mathrm{O}_{3} \mathrm{~S}^{+}-$ $\mathrm{ClO}_{4}^{-}$requires: $\mathrm{C}, 51.27 ; \mathrm{H}, 5.46 ; \mathrm{N}, 13.80 \%$.

l- $N$-[ (4-Guanidinoaminosulfonyl-phenyl) aminocarbonylpropyl]-2,6-di-npropyl-4-phenylpyridinium perchlorate, B6 As white crystals, m.p. $234-5^{\circ} \mathrm{C}$ (yield of $45 \%$ ). IR (KBr), $\mathrm{cm}^{-1}: 625,685,775,1100,1172,1285,1345,1540$, 1580, 1645, 1675, 3050, 3255, 3360; ${ }^{1} \mathrm{H}-\mathrm{NMR}$ (TFA), $\delta$, ppm: 1.23 (t, 6H, 2 Me from $\mathrm{Pr}$ ), 1.90 (m, 2H, $\beta$ - $\mathrm{CH}_{2}$ of GABA moiety), 2.28 ( $\mathrm{t}, 2 \mathrm{H}, \alpha-\mathrm{CH}_{2}$ of GABA moiety), 2.03 (q, 4H, $2 \mathrm{CH}_{2}$ from $\mathrm{Pr}$ ), 3.01 (t, $2 \mathrm{H}, \gamma-\mathrm{CH}_{2}$ of GABA moiety), 3.12-3.56 (m, 4H, $2 \mathrm{CH}_{2}$ from $\mathrm{Pr}$ ), 7.55-8.62 (m, 11H, ArH from 1,4-phenylene, 4-Ph and 3,5-H from pyridinium). Found: $\mathrm{C}, 52.71 ; \mathrm{H}, 5.60$; $\mathrm{N}, 13.05 . \mathrm{C}_{28} \mathrm{H}_{37} \mathrm{~N}_{6} \mathrm{O}_{3} \mathrm{~S}^{+} \mathrm{ClO}_{4}^{-}$requires: $\mathrm{C}, 52.78 ; \mathrm{H}, 5.85 ; \mathrm{N}, 13.19 \%$.

l-N-[(4-Guanidinoaminosulfonyl-phenyl) aminocarbonylpropyl]-2,6-di-isopropyl-4-phenylpyridinium perchlorate, $\mathbf{B} 7$ As white crystals, m.p. $241-3^{\circ} \mathrm{C}$ (yield of 47\%). IR (KBr), $\mathrm{cm}^{-1}: 625,685,765,1100,1174,1285,1345,1540$, $1580,1645,1675,3060,3270,3355 ;{ }^{1} \mathrm{H}-\mathrm{NMR}$ (TFA), $\delta$, ppm: 1.60 (d, 12H, 4 Me from $i$-Pr), $1.90\left(\mathrm{~m}, 2 \mathrm{H}, \beta-\mathrm{CH}_{2}\right.$ of GABA moiety), $2.28\left(\mathrm{t}, 2 \mathrm{H}, \alpha-\mathrm{CH}_{2}\right.$ of GABA moiety), 3.01 (t, $2 \mathrm{H}, \gamma-\mathrm{CH}_{2}$ of GABA moiety), 3.10-3.62 (m, $2 \mathrm{H}, 2$ $\mathrm{CH}$ from $i$ - $\mathrm{Pr}), 7.47-8.43(\mathrm{~m}, 11 \mathrm{H}, \mathrm{ArH}$ from 1,4-phenylene, 4- $\mathrm{Ph}$ and 3,5$\mathrm{H}$ from pyridinium). Found: $\mathrm{C}, 52.62 ; \mathrm{H}, 5.92 ; \mathrm{N}, 13.13 . \mathrm{C}_{28} \mathrm{H}_{37} \mathrm{~N}_{6} \mathrm{O}_{3} \mathrm{~S}^{+}$$\mathrm{ClO}_{4}^{-}$requires: $\mathrm{C}, 52.78 ; \mathrm{H}, 5.85 ; \mathrm{N}, 13.19 \%$.

I-N-[(4-Guanidinoaminosulfonyl-phenyl) aminocarbonylpropyl]-2-methyl4,6-diphenylpyridinium perchlorate, $\mathbf{B 8}$ As white crystals, m.p. $233-4^{\circ} \mathrm{C}$ (yield of 19\%). IR (KBr), cm ${ }^{-1}: 625,675,775,1100,1173,1285,1345,1540$, $1580,1645,1675,3050,3240,3365$; ${ }^{1} \mathrm{H}-\mathrm{NMR}$ (TFA), $\delta$, ppm: $1.90(\mathrm{~m}, 2 \mathrm{H}$, $\beta-\mathrm{CH}_{2}$ of GABA moiety), 2.28 (t, $2 \mathrm{H}, \alpha-\mathrm{CH}_{2}$ of GABA moiety), 3.03 ( $\mathrm{t}, 2 \mathrm{H}$, $\gamma-\mathrm{CH}_{2}$ of GABA moiety), 3.30 (s, 3H, 2-Me), 7.13-8.49 (m, 16H, ArH from 1,4-phenylene, 4,6- $\mathrm{Ph}_{2}$ and 3,5-H from pyridinium). Found: $\mathrm{C}, 54.49$; $\mathrm{H}, 4.62 ; \mathrm{N}, 13.00 . \mathrm{C}_{29} \mathrm{H}_{31} \mathrm{~N}_{6} \mathrm{O}_{3} \mathrm{~S}^{+} \mathrm{ClO}_{4}^{-}$requires: $\mathrm{C}, 54.16 ; \mathrm{H}, 4.86 ; \mathrm{N}$, $13.07 \%$.

I-N-[(4-Guanidinoaminosulfonyl-phenyl) aminocarbonylpropyl]-2-ethyl-4,6diphenylpyridinium perchlorate, B9 As white crystals, m.p. $235-7^{\circ} \mathrm{C}$ (yield of $24 \%$ ). IR (KBr), $\mathrm{cm}^{-1}: 625,685,750,1100,1173,1285,1345,1540$, $1580,1645,1675,3050,3225,3360 ;{ }^{1} \mathrm{H}-\mathrm{NMR}$ (TFA), $\delta$, ppm: $1.72(\mathrm{t}, 3 \mathrm{H}, \mathrm{Me}$ from Et), $1.90\left(\mathrm{~m}, 2 \mathrm{H}, \beta-\mathrm{CH}_{2}\right.$ of GABA moiety), $2.28\left(\mathrm{t}, 2 \mathrm{H}, \alpha-\mathrm{CH}_{2}\right.$ of GABA moiety), $3.01\left(\mathrm{t}, 2 \mathrm{H}, \gamma-\mathrm{CH}_{2}\right.$ of GABA moiety), 3.21-3.78 (m, $2 \mathrm{H}$, $\mathrm{CH}_{2}$ from Et), 7.21-8.54 (m, 16H, ArH from 1,4-phenylene, 4,6- $\mathrm{Ph}_{2}$ and 
3,5-H from pyridinium). Found: $\mathrm{C}, 54.92 ; \mathrm{H}, 4.98 ; \mathrm{N}, 12.67 . \mathrm{C}_{30} \mathrm{H}_{33} \mathrm{~N}_{6} \mathrm{O}_{3} \mathrm{~S}^{+}-$ $\mathrm{ClO}_{4}^{-}$requires: $\mathrm{C}, 54.83 ; \mathrm{H}, 5.06 ; \mathrm{N}, 12.79 \%$.

l-N-[(4-Guanidinoaminosulfonyl-phenyl) aminocarbonylpropyl]-2-n-propyl4,6-diphenylpyridinium perchlorate, B10 As white crystals; m.p. $230-2^{\circ} \mathrm{C}$ (yield of 32\%). IR (KBr), $\mathrm{cm}^{-1}: 625,705,775,1100,1174,1285,1345,1540$, $1580,1645,1675,3080,3255,3360 ;{ }^{1} \mathrm{H}-\mathrm{NMR}$ (TFA), $\delta, \mathrm{ppm}: 1.32(\mathrm{t}, 3 \mathrm{H}, \mathrm{Me}$ from $\mathrm{Pr}$ ), 1.90 (m, 2H. $\beta-\mathrm{CH}_{2}$ of GABA moiety), 2.28 (t, $2 \mathrm{H}, \alpha-\mathrm{CH}_{2}$ of GABA moiety), 2.17 (sextet, $2 \mathrm{H}, \mathrm{CH}_{2}$ from $n$-Pr), $3.00\left(\mathrm{t}, 2 \mathrm{H}, \gamma-\mathrm{CH}_{2}\right.$ of GABA moiety), $3.12-3.60\left(\mathrm{~m}, 2 \mathrm{H}, \mathrm{CH}_{2}\right.$ from $n$-Pr), 7.12-8.38 (m, $16 \mathrm{H}$, ArH from 1,4-phenylene, 4,6- $\mathrm{Ph}_{2}$ and $3,5-\mathrm{H}$ from pyridinium). Found: $\mathrm{C}$, $55.34 ; \mathrm{H}, 5.30 ; \mathrm{N}, 12.38 . \mathrm{C}_{31} \mathrm{H}_{35} \mathrm{~N}_{6} \mathrm{O}_{3} \mathrm{~S}^{+} \mathrm{ClO}_{4}^{-}$requires: $\mathrm{C}, 55.48 ; \mathrm{H}, 5.26 ; \mathrm{N}$, $12.52 \%$.

1-N-[(4-Guanidinoaminosulfonyl-phenyl) aminocarbonylpropyl]-2-iso-propyl-4,6-dipheny/pyridinium perchlorate, B11 As white crystals, m.p. 234$5^{\circ} \mathrm{C}$ (yield of $36 \%$ ). IR ( $\mathrm{KBr}$ ), $\mathrm{cm}^{-1}: 625,700,765,1100,1175,1280,1345$, $1540,1580,1645,1675,3070,3250.3365 ;{ }^{1} \mathrm{H}-\mathrm{NMR}$ (TFA), $\delta$, ppm: 1.70 (d, $6 \mathrm{H}, 2 \mathrm{Me}$ from $i-\mathrm{Pr}), 1.90\left(\mathrm{~m}, 2 \mathrm{H}, \beta-\mathrm{CH}_{2}\right.$ of GABA moiety), $2.28(\mathrm{t}, 2 \mathrm{H}$, $\alpha-\mathrm{CH}_{2}$ of GABA moiety), 3.01 ( $\mathrm{t}, 2 \mathrm{H}, \gamma-\mathrm{CH}_{2}$ of GABA moiety), 3.50-3.82 (m, $1 \mathrm{H}, \mathrm{CH}$ from $i-\mathrm{Pr}), 7.21-8.50(\mathrm{~m}, 16 \mathrm{H}$, ArH from 1,4-phenylene, 4,6$\mathrm{Ph}_{2}$ and 3,5-H from pyridinium). Found: $\mathrm{C}, 55.32 ; \mathrm{H}, 5.16 ; \mathrm{N}, 12.45$. $\mathrm{C}_{31} \mathrm{H}_{35} \mathrm{~N}_{6} \mathrm{O}_{3} \mathrm{~S}^{+} \mathrm{ClO}_{4}^{-}$requires: $\mathrm{C}, 55.48 ; \mathrm{H}, 5.26 ; \mathrm{N}, 12.52 \%$.

1-N-[ (4-Guanidinoaminosulfonyl-phenyl) aminocarbonylpropyl]-2-n-butyl4,6-diphenylpyridinium perchlorate, B12 As white crystals, m.p. $208-9^{\circ} \mathrm{C}$ (yield of $51 \%$ ). IR $(\mathrm{KBr}), \mathrm{cm}^{-1}: 625,685,765,1100,1175,1285,1345,1540$, $1580,1645,1675,3080,3255,3355 ;{ }^{1} \mathrm{H}-\mathrm{NMR}$ (TFA), $\delta, \mathrm{ppm}: 1.15$ (t, 3H, Me from $n-\mathrm{Bu}), 1.38-2.45\left(\mathrm{~m}, 8 \mathrm{H}, 2 \mathrm{CH}_{2}\right.$ from $n-\mathrm{Bu}+\beta-\mathrm{CH}_{2}$ of GABA moiety $+\alpha-\mathrm{CH}_{2}$ of GABA moiety), 3.01 (t, $2 \mathrm{H}, \gamma-\mathrm{CH}_{2}$ of GABA moiety), 3.10-3.69 $\left(\mathrm{m}, 2 \mathrm{H}, \mathrm{CH}_{2}\right.$ from $\left.n-\mathrm{Bu}\right), 7.21-8.49(\mathrm{~m}, 16 \mathrm{H}, \mathrm{ArH}$ from $1,4-$ phenylene; $4,6-\mathrm{Ph}_{2}$ and 3,5-H from pyridinium). Found: $\mathrm{C}, 55.99 ; \mathrm{H}, 5.64$; $\mathrm{N}, 12.15 . \mathrm{C}_{32} \mathrm{H}_{37} \mathrm{~N}_{6} \mathrm{O}_{3} \mathrm{~S}^{+} \mathrm{ClO}_{4}^{-}$requires: $\mathrm{C}, 56.09 ; \mathrm{H}, 5.44 ; \mathrm{N}, 12.26 \%$.

I-N-[ /4-Guanidinoaminosulfonyl-phenyl) aminocarbonylpropyl]-2-tert-butyl4,6-diphenylpyridinium perchlorate, B13 As white crystals, m.p. $193-4^{\circ} \mathrm{C}$ (yield of $60 \%$ ). IR (KBr), $\mathrm{cm}^{-1}: 625,700,765,1100,1173,1285,1345$, 1540, 1580, 1645, 1675, 3060,3250,3360; 'H-NMR (TFA), $\delta$, ppm: 1.85 (s. $9 \mathrm{H}, t-\mathrm{Bu}), 1.91\left(\mathrm{~m}, 2 \mathrm{H}, \beta-\mathrm{CH}_{2}\right.$ of GABA moiety), $2.28\left(\mathrm{t}, 2 \mathrm{H}, \alpha-\mathrm{CH}_{2}\right.$ of GABA moiety), 3.01 (t, $2 \mathrm{H}, \gamma-\mathrm{CH}_{2}$ of GABA moiety), $6.90-8.79(\mathrm{~m}, 16 \mathrm{H}$, ArH from 1,4-phenylene, 4,6- $\mathrm{Ph}_{2}$ and 3,5- $\mathrm{H}$ from pyridinium). Found: $\mathrm{C}$, $56.09 ; \mathrm{H}, 5.35: \mathrm{N}, 12.21 . \mathrm{C}_{32} \mathrm{H}_{37} \mathrm{~N}_{6} \mathrm{O}_{3} \mathrm{~S}^{+} \mathrm{ClO}_{4}^{-}$requires: $\mathrm{C}, 56.09 ; \mathrm{H}, 5.44 ; \mathrm{N}$, $12.26 \%$. 
1-N-[(4-Guanidinoaminosulfonyl-phenyl) aminocarbonylpropyl]-2,4,6-triphenylpyridinium perchlorate, B14 As yellow crystals, m.p. $203-4^{\circ} \mathrm{C}$ (yield of $71 \%$ ). IR (KBr), $\mathrm{cm}^{-1}: 625,680,770,1100,1175,1285,1345,1540,1580$, $1645,1675,3050,3260,3365 ;{ }^{1} \mathrm{H}-\mathrm{NMR}$ (TFA), $\delta$, ppm: $1.90\left(\mathrm{~m}, 2 \mathrm{H}, \beta-\mathrm{CH}_{2}\right.$ of GABA moiety), $2.28\left(\mathrm{t}, 2 \mathrm{H}, \alpha-\mathrm{CH}_{2}\right.$ of GABA moiety), $3.01\left(\mathrm{t}, 2 \mathrm{H}, \gamma-\mathrm{CH}_{2}\right.$ of GABA moiety), 6.71-8.49 (m, 21H, ArH from 1,4-phenylene, 2,4,6- $\mathrm{Ph}_{3}$ and 3,5-H from pyridinium). Found: $\mathrm{C}, 57.63 ; \mathrm{H}, 4.87 ; \mathrm{N}, 11.76$. $\mathrm{C}_{34} \mathrm{H}_{33} \mathrm{~N}_{6} \mathrm{O}_{3} \mathrm{~S}^{+} \mathrm{ClO}_{4}^{-}$requires: $\mathrm{C}, 57.91 ; \mathrm{H}, 4.72 ; \mathrm{N}, 11.92 \%$.

l-N-[(4-Guanidinoaminosulfonyl-phenyl) aminocarbonylpropyl]-2,6-diphenylpyridinium perchlorate, B15 As yellow crystals, m.p. $221-3^{\circ} \mathrm{C}$ (yield of $32 \%)$. IR (KBr), cm $\mathrm{cm}^{-1}: 625,700,760,1100,1176,1285,1345,1540,1580$, $1645,1675,3050,3240,3365 ;{ }^{1} \mathrm{H}-\mathrm{NMR}$ (TFA), $\delta$, ppm: $1.90\left(\mathrm{~m}, 2 \mathrm{H}, \beta-\mathrm{CH}_{2}\right.$ of GABA moiety), $2.28\left(\mathrm{t}, 2 \mathrm{H}, \alpha-\mathrm{CH}_{2}\right.$ of GABA moiety), $3.01\left(\mathrm{t}, 2 \mathrm{H}, \gamma-\mathrm{CH}_{2}\right.$ of GABA moiety), 6.89-8.50 (m, 17H, ArH from 1,4-phenylene, 2,6- $\mathrm{Ph}_{2}$ and $3,4,5-\mathrm{H}$ from pyridinium). Found: $\mathrm{C}, 53.60 ; \mathrm{H}, 4.41 ; \mathrm{N}, 13.24$. $\mathrm{C}_{28} \mathrm{H}_{29} \mathrm{~N}_{6} \mathrm{O}_{3} \mathrm{~S}^{+} \mathrm{ClO}_{4}^{-}$requires: $\mathrm{C}, 53.46 ; \mathrm{H}, 4.65 ; \mathrm{N}, 13.36 \%$.

l-N-[(4-Guanidinoaminosulfonyl-phenyl) aminocarbonylpropyl]-2,3,4,6-tetramethylpyridinium perchlorate, B16 As white crystals, m.p. $217-9^{\circ} \mathrm{C}$ (yield of $62 \%)$. IR (KBr), $\mathrm{cm}^{-1}: 625,680,1100,1172,1285,1345,1540,1580,1645$, $1675,3030,3245,3365 ;{ }^{1} \mathrm{H}-\mathrm{NMR}$ (TFA), $\delta, \mathrm{ppm}: 1.90\left(\mathrm{~m}, 2 \mathrm{H}, \beta-\mathrm{CH}_{2}\right.$ of GABA moiety), $2.28\left(\mathrm{t}, 2 \mathrm{H}, \alpha-\mathrm{CH}_{2}\right.$ of GABA moiety), 2.52 (s, 3H, 3-Me); 2.62 (s, 3H, 4-Me), 2.87 (s, 3H, 6-Me), 2.91 (s, 3H, 2-Me), 3.04 (t, 2H, $\gamma-$ $\mathrm{CH}_{2}$ of GABA moiety), 7.55-8.49 (m, 5H, ArH from 1,4-phenylene $+5-\mathrm{H}$ from pyridinium). Found: $\mathrm{C}, 44.92 ; \mathrm{H}, 5.46 ; \mathrm{N}, 15.69 . \mathrm{C}_{20} \mathrm{H}_{29} \mathrm{~N}_{6} \mathrm{O}_{3} \mathrm{~S}^{+} \mathrm{ClO}_{4}^{-}$ requires: $\mathrm{C}, 45.07 ; \mathrm{H}, 5.48 ; \mathrm{N}, 15.77 \%$.

\section{Enzyme Assays: $K_{1}$ Determinations}

Human thrombin and human trypsin were purchased from Sigma Chemical Co. (St. Louis, MO, USA); their concentrations were determined from the absorbance at $280 \mathrm{~nm}$ and the extinction coefficients furnished by the supplier. The activity of such preparations was in the range of $2500-3000 \mathrm{NIH}$ units/mg. The potency of standard and novel inhibitors was determined from the inhibition of the enzymatic (amidolytic) activity of these serine proteases, at $21^{\circ} \mathrm{C}$, using Ts-Gly-Pro-Arg-pNA (Chromozym TH) as substrate, by the method of Lottenberg et al. ${ }^{23}$ The substrate was reconstituted as $4 \mathrm{mM}$ stock in ultrapure water and brought to $\mathrm{pH} 4$ with hydrochloric acid. Substrate concentrations were determined from absorbance at the isosbestic wavelength for the peptide- $p$-nitroanilide-p-nitroaniline mixtures. 
Extinction coefficients of $8270 \mathrm{~L} \mathrm{~mol}^{-1} \mathrm{~cm}^{-1}$ in the buffer used $(0.01 \mathrm{M}$ Hepes $-0.01 \mathrm{M}$ Tris $-0.1 \mathrm{M} \mathrm{NaCl}-0.1 \%$ polyethylene glycol 6000 ) were employed. The rate of $p$-nitroanilide hydrolysis was determined from the change in absorbance at $405 \mathrm{~nm}$ using an extinction coefficient for $p$-nitroaniline of $9920 \mathrm{~L} \mathrm{~mol}^{-1} \mathrm{~cm}^{-1}$ in the above-mentioned reaction buffer. Measurements were made using a Cart 3 spectrophotometer interfaced with a PC. Initial velocities were thus estimated using the direct linear plot-based procedure as reported by Lottenberg et al ${ }^{23} K_{\mathrm{I}}$ values were then determined according to Dixon, using a linear regression program. ${ }^{24}$ The $K_{\mathrm{I}}$ values determined are the means of at least three determinations.

\section{Calculations}

Calculations were done with Gaussian $94^{25}$ and MOPAC $93 .{ }^{26}$ The two tautomeric structures of phenylsulfonyl-aminoguanidine, 11A and 11B, were generated using Hyperchem $5.1^{27}$ and optimized using MM+. These geometries were further optimized with MOPAC 93 using the AM1 Hamiltonian. ${ }^{28}$ The geometries thus obtained were used in one point calculations to obtain the heats of formation in vacuo and in a medium of dielectric constant 72.7 using the $\operatorname{COSMO}^{29}$ approximation (by MOPAC), and with the B3LYP method ${ }^{30.31}$ and in the latter medium using SCIPCM ${ }^{32}$ (by Gaussian). $\mathrm{p} K_{\mathrm{a}}$ values were calculated using the Pallas software. ${ }^{33}$

\section{RESULTS AND DISCUSSION}

The lead molecule for obtaining novel types of thrombin inhibitors considered by us was benzamidine which possesses an inhibition constant $K_{\mathrm{I}}=300 \mathrm{nM}$ against human thrombin; moreover, the $\mathrm{X}$-ray crystallographic structure for the complex of benzamidine with this enzyme was reported some time ago (PDB entry: 1DWB) ${ }^{34}$ From the X-ray data it was observed that the amidino moiety of the inhibitor is anchored to the S1 specificity pocket of the enzyme, interacting electrostatically and by means of hydrogen bonds with Asp 189. Several other van der Waals contacts between the inhibitor molecule and the enzyme were also seen. ${ }^{34}$ Obviously, benzamidine is a weak thrombin inhibitor, since the binding energy is mainly gained due to the strong electrostatic interaction of the carboxylate of Asp 189 and the positively charged amidino moiety. However, as already mentioned in the introductory section, the amidino moiety possesses too high a basicity to allow the formation of bioavailable enzyme inhibitors, and it appeared thus 
of great interest to elaborate weakly basic variants of this attractive thrombin anchoring group. The sulfonyl-aminoguanidino moiety appeared as an attractive candidate for such a purpose, since the presence of the $\mathrm{SO}_{2}$ moiety in the neighborhood of the strong base, aminoguanidine, should drastically weaken its basicity. Such a modified anchoring group should not presumably interfere with the binding of the inhibitor to the enzyme, since the hydrogen-bonding donor/acceptor properties as well as the possibility to interact electrostatically with the enzyme for the compounds incorporating this group should not differ too much from those of the classical amidino- $/$ guanidino-based inhibitors of types 1-5 or 10. Since sulfonyl-aminoguanidines possesses a large number of possible tautomeric forms, and this factor might be a critical one for binding to thrombin, we performed MOPAC as well as AM1 calculations in order to identify the most stable tautomers. As seen from data of Table I, the tautomer of type 11A is less stable than the tautomer 11B (Scheme 1) in the case of benzenesulfonyl-aminoguanidine (Scheme 1). Moreover, $\mathrm{p} K_{\mathrm{a}}$ calculations showed that this compound is protonatable with a $\mathrm{p} K_{\mathrm{a}}$ of 6.84 , which might be very favorable for the bioavailability and other pharmacological properties of this type of putative thrombin inhibitors (Table I).

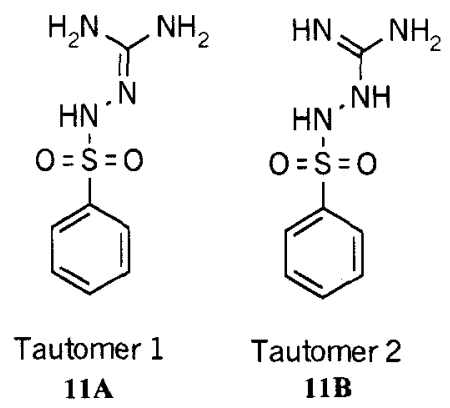

SCHEME 1 Possible tautomers of phenylsulfonyl-aminoguanidine 11.

TABLE I Calculated parameters for the tautomers 11A and 11B of benzenesulfonyl-aminoguanidine (see Scheme 1)

\begin{tabular}{lccccc}
\hline Tautomer & AM1/Vacuum & AM1/COSMO & DF/Vacuum & DF/SCIPCM & p $K_{\mathrm{a}}$ Pallas \\
\hline $\mathbf{1 1 A}$ & 0.47 & -38.42 & -1040.254819 & -1040.278149 & $*$ \\
11B & 10.95 & -28.77 & -1040.262145 & -1040.282850 & 6.84 \\
Difference & $-\mathbf{1 0 . 4 8}$ & $\mathbf{- 9 . 6 5}$ & $+\mathbf{4 . 6 0}$ & $+\mathbf{2 . 9 5}$ & - \\
$(\mathbf{1 1 A}-\mathbf{1 1 B})$ & & & & & \\
\hline
\end{tabular}

$\left(\mathrm{DF}=\mathrm{B} 3 \mathrm{LYP} / 6-31 \mathrm{G}^{*} / \mathrm{AM} 1\right)$ The AMl results are in kcal and the density functional results in Hartrees, except for the differences (in bold) which are in kcal throughout.

${ }^{*}$ No acidic or basic groups found (no $\mathrm{p} K_{\mathrm{a}}$ ) between -20 and +50 . 
Thus, a first series of sulfonyl-aminoguanidines $\mathbf{A 1} \mathbf{- A 3 2}$ was prepared in order to test the above-mentioned hypothesis (Table II). These compounds were obtained by the simple reactions of alkyl-/aralkyl-/aryl-/hetarylsulfonyl halides or sulfonic acids anhydrides with nucleophiles. ${ }^{17,18}$

The sulfanilyl derivative $\mathbf{A 1 4}$ (one of the best thrombin inhibitors among the obtained compounds, see discussion later in the text) was selected for

TABLE II Inhibition of human thrombin and human trypsin by the sulfonyl-aminoguanidines A1-A32

$$
\mathrm{R}-\mathrm{SO}_{2} \mathrm{NHNHC}(=\mathrm{NH}) \mathrm{NH}_{2}
$$

A1-A32

\begin{tabular}{|c|c|c|c|c|}
\hline \multirow[t]{2}{*}{ Compound $\mathbf{A}$} & \multirow[t]{2}{*}{$R$} & \multicolumn{2}{|c|}{$K_{1}(\mathrm{nM})^{\mathrm{a}}$} & \multirow[t]{2}{*}{ Synthetic method } \\
\hline & & Thrombin & Trypsin & \\
\hline 1 & $\mathrm{Me}_{2} \mathrm{~N}$ & 1160 & 2800 & A \\
\hline 2 & $\mathrm{PhCH}_{2}-$ & 325 & 1290 & B \\
\hline 3 & $\mathrm{CF}_{3-}-$ & 800 & 1320 & $\mathrm{C}$ \\
\hline 4 & $p-\mathrm{F}-\mathrm{C}_{6} \mathrm{H}_{4}-$ & 225 & 1025 & A \\
\hline 5 & $p-\mathrm{Cl} \sim \mathrm{C}_{6} \mathrm{H}_{4}-$ & 212 & 1100 & A \\
\hline 6 & $p-\mathrm{Br}-\mathrm{C}_{6} \mathrm{H}_{4}-$ & 203 & 1215 & A \\
\hline 7 & $p-\mathrm{I} \cdots \mathrm{C}_{6} \mathrm{H}_{4}-$ & 177 & 1300 & A \\
\hline 8 & $p-\mathrm{CH}_{3}-\mathrm{C}_{6} \mathrm{H}_{4}$ & 270 & 1775 & A \\
\hline 9 & $p-\mathrm{O}_{2} \mathrm{~N}-\mathrm{C}_{6} \mathrm{H}_{4}$ & 166 & 990 & A \\
\hline 10 & $m-\mathrm{O}_{2} \mathrm{~N}-\mathrm{C}_{6} \mathrm{H}_{4}$ & 170 & 1235 & A \\
\hline 11 & $o-\mathrm{O}_{2} \mathrm{~N} \quad \mathrm{C}_{6} \mathrm{H}_{4}$ & 324 & 1800 & A \\
\hline 12 & $3-\mathrm{Cl}-4-\mathrm{O}_{2} \mathrm{~N}-\mathrm{C}_{6} \mathrm{H}_{3}^{--}$ & 154 & 1010 & A \\
\hline 13 & $p-\mathrm{AcNH}-\mathrm{C}_{6} \mathrm{H}_{4}-$ & 172 & 1025 & A \\
\hline 14 & $p-\mathrm{H}_{2} \mathrm{~N}-\mathrm{C}_{6} \mathrm{H}_{4}-$ & 91 & 1425 & B \\
\hline 15 & $m-\mathrm{H}_{2} \mathrm{~N}-\mathrm{C}_{6} \mathrm{H}_{4}-$ & 88 & 1400 & B \\
\hline 16 & $\mathrm{C}_{6} \mathrm{~F}_{5}$ & 123 & 1350 & A \\
\hline 17 & $0-\mathrm{HOOC}-\mathrm{C}_{6} \mathrm{H}_{4-}^{-}$ & 205 & 1400 & $\mathrm{D}$ \\
\hline 18 & $m-\mathrm{HOOC}-\mathrm{C}_{6} \mathrm{H}_{4}-$ & 112 & 1520 & A \\
\hline 19 & $p-\mathrm{HOOC}-\mathrm{C}_{6} \mathrm{H}_{4}-$ & 97 & 1335 & A \\
\hline 20 & $0-\mathrm{HOOC}-\mathrm{C}_{6} \mathrm{Br}_{4}-$ & 213 & 1200 & $\mathrm{D}$ \\
\hline 21 & $p-\mathrm{CH}_{3} \mathrm{O}-\mathrm{C}_{6} \mathrm{H}_{4}^{-}$ & 227 & 1275 & A \\
\hline 22 & $2.4 .6-\left(\mathrm{CH}_{3}\right)_{3}-\mathrm{C}_{6} \mathrm{H}_{2} \ldots$ & 219 & 1345 & A \\
\hline 23 & $4-\mathrm{CH}_{3} \mathrm{O}-3-\mathrm{H}_{2} \mathrm{~N}-\mathrm{C}_{6} \mathrm{H}_{3}$ & 98 & 1100 & B \\
\hline 24 & $2-\mathrm{HO}-3,5-\mathrm{Cl}_{2}-\mathrm{C}_{6} \mathrm{H}_{2}--$ & 139 & 1355 & A \\
\hline 25 & $4-\mathrm{Me}_{2} \mathrm{~N} \cdot \mathrm{C}_{6} \mathrm{H}_{4}-\mathrm{N}=\mathrm{N}-\mathrm{C}_{6} \mathrm{H}_{4}-$ & 130 & 1200 & A \\
\hline 26 & 5-Dimethylamino-1-naphthyl- & 114 & 1350 & A \\
\hline 27 & 1-Naphthyl & 125 & 1200 & A \\
\hline 28 & 2-Naphthyl & 129 & 1285 & A \\
\hline 29 & $n-\mathrm{C}_{4} \mathrm{~F}_{9}-$ & 500 & 2055 & A \\
\hline 30 & $n-\mathrm{C}_{8} \mathrm{~F}_{17}$ & 335 & 1920 & A \\
\hline 31 & 2-thienyl & 170 & 1250 & A \\
\hline 32 & Camphor-10-yl & 305 & 990 & A \\
\hline$\cdots \cdots$ & Benzamidine & 300 & 450 & - \\
\hline
\end{tabular}

Synthetic methods. A: $\mathrm{RSO}_{2} \mathrm{Cl}+$ aminoguanidine: $\mathrm{B}: \mathrm{RSO}_{2} \mathrm{~F}+$ aminoguanidine: $\mathrm{C}$ : triflic anhydride + aminoguanidine: $\mathrm{D}$ : sulfobenzoic cyclic anhydride + aminoguanidine.

${ }^{2} K_{1}$ values were obtained fron Dixon plots using a linear regression program. from at least three different assays. Spreads around the mean (data not shown) were $\pm 5-10 \%$ of the shown values. 
further elaboration, with the aim of obtaining more potent inhibitors. Two main approaches were used for attaining this purpose: (1) attachment of protected amino acyl/dipeptidyl moieties to the N-4 atom of the lead A14, and (2) attachment of substituted-pyridinium-propylcarboxamido moieties to the same $\mathrm{N}-4$ atom of the lead.

The data in Table II show that the sulfonyl-aminoguanidines A1-A32 possess weak thrombin inhibitory properties, comparable with those of the lead molecule, benzamidine. The main difference between the two classes of inhibitors regards the decreased affinity of our compounds for trypsin, as compared to benzamidine. The data also clearly show that the presence of an aromatic/heterocyclic ring in the molecule of such a compound highly enhances its affinity for thrombin. The aliphatic compounds, such as the dimethylamino-derivatives $\mathbf{A} \mathbf{1}$, or the perfluoroalkyl sulfonyl-aminoguanidines A3, A29, and $\mathbf{A 3 0}$ were among the most ineffective thrombin/trypsin inhibitors in the whole series of prepared compounds, with lower affinities than benzamidine 9 for the thrombin. The derivatives of the substitutedbenzenesulfonyl-aminoguanidine on the other hand possessed generally a higher affinity for thrombin, as compared to benzamidine. Typically, they inhibited thrombin with $K_{\mathrm{I}}$ values between 90 and $300 \mathrm{nM}$, but possessed a lower affinity for trypsin as compared to benzamidine, which is a highly desirable feature for an inhibitor to be developed for clinical applications. Moieties leading to effective thrombin inhibitors were: $p$-halogeno-phenyl, $p$-nitrophenyl, 3-chloro-4-nitrophenyl, $p$ - and $m$-amino-phenyl, perfluorophenyl, $p$-carboxy-phenyl, 4-methoxy-3-amino-phenyl, 1- and 2-naphthyl or 2-thienyl among others. In the new series of compounds, the best thrombin inhibitors proved to be those containing $p$ - or $m$-amino moieties. Thus, one such compound, sulfanilyl-aminoguanidine A14, was chosen as lead for further elaboration with the aim of obtaining more efficient inhibitors.

The first approach mentioned above is based primarily on the well-known scaffold Phe-Pro-Arg, which has been shown to assure effective binding of compounds containing it within the thrombin active site. ${ }^{3-5,7,35}$ Since our compounds already possessed the S1 anchoring moiety, basically Phe and Pro substitutions were performed initially. The terminal-amino moiety of these amino acids was protected by means of the classical benzyloxycarbonyl group (Cbz) or by the new tosylureido (ts) group, which has not been previously used for the design of serine protease inhibitors. Our choice was motivated by the following facts: the toluenesulfonyl group contained in this moiety might participate in interactions with the hydrophobic moieties of the thrombin binding site (such as S2 or/and S4, in the case of compounds possessing a larger molecule, see later in the text). Additionally, the 
$\mathrm{SO}_{2} \mathrm{NHCONH}$ part of the molecule might also be critical for binding, as in the X-ray structure of the NAPAP adduct of thrombin where it was observed that the $\mathrm{SO}_{2} \mathrm{NHCH}_{2} \mathrm{CO}$ moiety forms two strong hydrogen bonds with Gly $216 .{ }^{36}$ Thus, the dipeptidyl-derivatives ts- $D$-Phe-Pro- as well as Cbz-D-Phe-Pro- of the two leads were prepared (compounds A36, A37), and proved to be the most potent inhibitors in the whole series, together with A39. Also considering the fact that the well-known serine protease substrate Chromozym TH (Ts-Gly-Pro-Arg-p-nitroanilide; Ts $=4$-toluenesulfonyl) it possesses a strong affinity for thrombin $\left(\mathrm{K}_{\mathrm{m}} 4-12 \mu \mathrm{M}\right)^{23}$ we decided to use part of this scaffold for designing sulfanilyl-aminoguanidine-based inhibitors. This scaffold was modified as follows: the tosyl (Ts) group was changed to the ts (tosylureido) one and the amino terminal Gly was either maintained (i.e. A38) or changed to 3-Ala (i.e. A39) in order to study the influence of the length of this part of the molecule to binding to the enzyme. Most importantly, the Pro of the original scaffold was changed to the much more polar His in the new compounds A38 and A39. These changes led to effective thrombin inhibitors (Table II). Finally, among the different other dipeptides attached to the two lead molecules mentioned above (data not shown), the one giving strong and selective thrombin inhibitors was that based on the ts-Pro-Gly scaffold, in derivative A40. No enzyme-inhibitor structural data are available up to now for explaining the high affinity of such inhibitors to thrombin.

The synthetic strategy for attaching the amino acyl moieties to the lead A14 is outlined in Scheme 2. Basically, the synthesis is based on our observation that the reaction between $N$-protected amino acid/dipeptides and sulfanilyl fluoride 6 (in the presence of carbodiimide derivatives, such as EDCI) is much more rapid than the corresponding sulfanilation reactions (that would lead to sulfanilyl-sulfanilamide derivatives). Thus, a one-pot procedure for the preparation of compounds A33-A40 has been developed. The $N$-protected amino acid/dipeptide was treated with EDCI/triethyl amine in acetonitrile, then sulfanilyl fluoride 6 was added to give the benzenesulfonyl fluoride derivatives $\mathbf{8}$ which were not isolated but further transformed to the desired products by reaction with aminoguanidine. HPLC purification afforded compounds A33-A40 in good yields.

The second approach mentioned above took advantage of our observation that sulfanilyl-aminoguanidine $\mathbf{A 1 4}$ can be coupled with substitutedpyridinium-amino alkyl carboxylic acid derivatives, such as the GABA-derived compounds 9 , in the presence of carbodiimides preponderently at the $\mathrm{N}-4$ atom, without acylation of the aminoguanidine nitrogens, when working at $2-4^{\circ} \mathrm{C}$ (Scheme 3 ). 


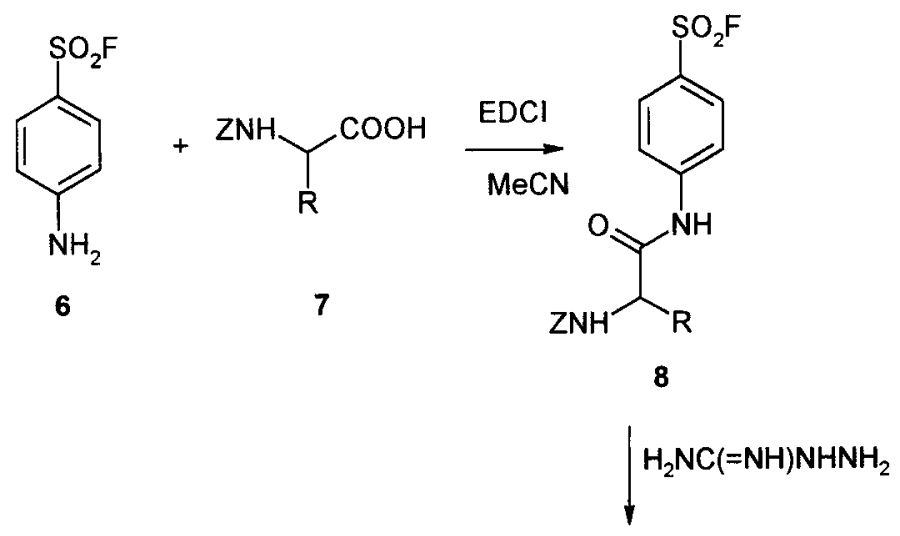

$\mathrm{Z}=\mathrm{Z}$-aminoacyl; $\mathrm{Cbz}$ or ts

$$
\begin{aligned}
& \mathrm{Cbz}=\mathrm{C}_{6} \mathrm{H}_{5} \mathrm{CH}_{2} \mathrm{OCO} \\
& \text { ts }=\mathrm{p}-\mathrm{Me}-\mathrm{C}_{6} \mathrm{H}_{4}-\mathrm{SO}_{2} \mathrm{NHCO}
\end{aligned}
$$<smiles></smiles>

A33-A40

SCHEME 2 Synthesis of compounds A33-A40.<smiles>N=C(N)NNS(=O)(=O)c1ccc(N)cc1</smiles>

A14<smiles>[R3]c1cc([R4])[n+](CCCC(=O)O)c([R1])c1[R2]</smiles>

9<smiles>[R2]C=C([R1])N(C=[R4])CCCC(=O)Nc1ccc(S(=O)(=O)NNC(=N)N)cc1</smiles>

SCHEME 3 Synthetic strategy of compounds B1-B16. 
Inhibition data against two serine proteases, human thrombin and human trypsin are shown in Tables II-IV for the compounds prepared here. ${ }^{23}$ Inhibition data with the standard inhibitors 1-3 are also provided for comparison.

In the subseries of amino acyl/dipeptidyl-containing inhibitors A33-A40, the following facts should be noted (Table III): (i) all these compounds behave as stronger thrombin inhibitors $\left(K_{\mathrm{I}}\right.$ values $\left.11-50 \mathrm{nM}\right)$ as compared to the lead from which they were obtained, whereas their affinity for trypsin remained relatively low ( $K_{\mathrm{I}}$ values of $1315-1560 \mathrm{nM}$ ). Furthermore, the affinity of the best inhibitors for thrombin is comparable or slightly better than that of the clinically used argatroban and inogatran although less effective than that of NAPAP. On the other hand, the thrombin:trypsin selectivity of our compounds is much better as compared to that of other reported inhibitors, (ii) protected-dipeptide derivatives (such as A36-A40) were more effective inhibitors as compared to the protected amino acyl derivatives A33-A35, (iii) the ts moiety seems to be slightly more beneficial than $\mathrm{Cbz}$ one for inhibitor potency towards thrombin and (iv) elongation of

TABLE III Inhibition of human thrombin and human trypsin by A33-A40 obtained from sulfanilyl-aminoguanidine A14 as lead. Inhibition data for the standard inhibitors $\mathbf{1 - 3}$ are also included

$$
p-\mathrm{XNH}_{-}-\mathrm{C}_{6} \mathrm{H}_{4}-\mathrm{SO}_{2} \mathrm{NHNHC}(=\mathrm{NH}) \mathrm{NH}_{2}
$$

A33-A40

\begin{tabular}{llcc}
\hline Compound & \multicolumn{1}{c}{$X^{*}$} & \multicolumn{2}{c}{$K_{1}(\mathrm{nM})^{\text {a }}$} \\
\cline { 3 - 4 } & & 50 & Thrombin \\
\hline A33 & Cbz-D-Phe & 44 & 1315 \\
$\mathbf{A 3 4}$ & ts- $D$-Phe & 43 & 1340 \\
$\mathbf{A 3 5}$ & ts- $L$-Pro & 10 & 1530 \\
$\mathbf{A 3 6}$ & ts- $D$-PhePro & 11 & 1450 \\
$\mathbf{A 3 7}$ & Cbz-D-PhePro & 15 & 1400 \\
$\mathbf{A 3 8}$ & ts-GlyHis & 10 & 1555 \\
$\mathbf{A 3 9}$ & ts-3-AlaHis & 15 & 1390 \\
$\mathbf{A 4 0}$ & ts- $L$-ProGly & 19 & 1560 \\
$\mathbf{1}$ & Argatroban & - \\
$\mathbf{2}$ & Inogatran & 15 & 540 \\
$\mathbf{3}$ & NAPAP & 6.5 & 690 \\
\hline
\end{tabular}

${ }^{a} K_{1}$ values were obtained fron Dixon plots using a linear regression program, from at least three different assays. Spreads around the mean (data not shown) werc $\pm 5-10^{\circ} \%$ of the shown values. ${ }^{b}$ From Ref. [5a]. ${ }^{*} \mathrm{Cbz}=\mathrm{PhCH}, \mathrm{OCO}$; $\mathrm{ts}=$ $p-\mathrm{MeC}_{6} \mathrm{H}_{4} \mathrm{SO}_{2} \mathrm{NHCO}-$ : these groups acylate the amino-terminal $\mathrm{H}_{2} \mathrm{~N}$ moiety. When configuration is not specified, $L$-amino acid moieties were employed. The usual polypeptide formalism is used: the amino-terminal residue is written first (and it is always protected either by the $\mathrm{Cbz}$ or the ts moiety), whereas the carboxy-terminal residue is acylating the sulfanilyl-aminoguanidine N-4 amino group. 
TABLE IV Inhibition of human thrombin and human trypsin by pyridiniumpropylcarboxamido-sulfanilyl-aminoguanidines (B1-B16)<smiles>[R3]c1cc([R4])[n+](CCCC(=O)Nc2ccc(S(=O)(=O)NNC(=N)N)cc2)c([R1])c1[R2]</smiles>

\begin{tabular}{|c|c|c|c|c|c|c|}
\hline \multirow[t]{2}{*}{ Compound } & \multirow[t]{2}{*}{$R^{l}$} & \multirow[t]{2}{*}{$R^{2}$} & \multirow[t]{2}{*}{$R^{3}$} & \multirow[t]{2}{*}{$R^{4}$} & \multicolumn{2}{|c|}{$K_{\mathrm{I}}(\mathrm{nM})^{\mathrm{a}}$} \\
\hline & & & & & Thrombin & Trypsin \\
\hline B1 & $\mathrm{Me}$ & $\mathrm{H}$ & $\mathrm{Me}$ & $\mathrm{Me}$ & 62 & 1150 \\
\hline B2 & $i-\operatorname{Pr}$ & $\mathrm{H}$ & $\mathrm{Me}$ & $\mathrm{Me}$ & 44 & 1100 \\
\hline B3 & $i-\operatorname{Pr}$ & $\mathrm{H}$ & $\mathrm{Me}$ & $i-\operatorname{Pr}$ & 71 & 1250 \\
\hline B4 & $\mathrm{Me}$ & $\mathrm{H}$ & $\mathrm{Ph}$ & $\mathrm{Me}$ & 24 & 1055 \\
\hline B5 & Et & $\mathrm{H}$ & $\mathrm{Ph}$ & Et & 23 & 1000 \\
\hline B6 & $n-\operatorname{Pr}$ & $\mathbf{H}$ & $\mathrm{Ph}$ & $n-\operatorname{Pr}$ & 42 & 1100 \\
\hline B7 & $i-\operatorname{Pr}$ & $\mathbf{H}$ & $\mathrm{Ph}$ & $i-\operatorname{Pr}$ & 40 & 1100 \\
\hline B8 & $\mathrm{Me}$ & $\mathrm{H}$ & $\mathrm{Ph}$ & $\mathrm{Ph}$ & 15 & 1100 \\
\hline B9 & $\mathrm{Et}$ & $\mathrm{H}$ & $\mathrm{Ph}$ & $\mathrm{Ph}$ & 11 & 1150 \\
\hline B10 & $n-\mathrm{Pr}$ & $\mathrm{H}$ & $\mathrm{Ph}$ & $\mathrm{Ph}$ & 18 & 1100 \\
\hline B11 & $i-\operatorname{Pr}$ & $\mathrm{H}$ & $\mathrm{Ph}$ & $\mathrm{Ph}$ & 17 & 1140 \\
\hline B12 & $n-\mathrm{Bu}$ & $\mathbf{H}$ & $\mathrm{Ph}$ & $\mathrm{Ph}$ & 39 & 1200 \\
\hline B13 & $t-\mathrm{Bu}$ & $\mathrm{H}$ & $\mathrm{Ph}$ & $\mathrm{Ph}$ & 12 & 1050 \\
\hline B14 & $\mathrm{Ph}$ & $\mathrm{H}$ & $\mathrm{Ph}$ & $\mathrm{Ph}$ & 40 & 1340 \\
\hline B15 & $\mathrm{Ph}$ & $\mathrm{H}$ & $\mathrm{H}$ & $\mathrm{Ph}$ & 43 & 1500 \\
\hline B16 & $\mathrm{Me}$ & $\mathrm{Me}$ & $\mathrm{Me}$ & $\mathrm{Me}$ & 62 & 1250 \\
\hline
\end{tabular}

${ }^{a} K_{I}$ values were obtained from Dixon plots using a linear regression program, from at least three different assays. Spreads around the mean (data not shown) were $\pm 5-10 \%$ of the shown values.

the molecule from Gly to $\beta$-Ala in compound A39 is also beneficial for its potency/selectivity profiles.

The following should be noted regarding the serine protease inhibition data for the positively-charged inhibitors B1-B16: (i) the pyridinium derivatives reported here generally behave as stronger thrombin inhibitors as compared to the lead molecules from which they were derived, i.e., sulfanilylaminoguanidine A14. At the same time, their affinity for trypsin is relatively low, which constitutes a positive feature for the putative clinical use of such a compounds, (ii) the nature of $\mathrm{R} 1-\mathrm{R} 4$ groups substituting the pyridinium 
ring was critical for the biological activity of the obtained compounds, similarly to the situation seen for the carbonic anhydrase sulfonamide inhibitors reported previously by our group. ${ }^{37.38}$ Thus, tri- or tetra-alkyl-pyridiniumas well as 2,6-di- or 2,4,6-triphenylpyridinium moieties were generally less effective than 2-alkyl-4,6-diphenyl-pyridinium groups in importing strong thrombin inhibitory properties to the compounds incorporating them. Practically. the most active derivatives were those containing 2-alkyl-4,6diphenyl-pyridinium moieties, such as 2-methyl-, 2-ethyl-, 2-iso-propyl-or 2-tert-butyl-4,6-diphenyl-pyridinium groups. Replacing the 2-alkyl group mentioned above with a bulky phenyl one (such as in B14) or with a longer aliphatic chain ( $n$-butyl, such as in B12) led to a drastic reduction in thrombin inhibitory potency. On the other hand, compounds possessing 2,6-dialkyl-4-phenyl-pyridinium moieties in their molecules (such as B4, B5) possessed a behaviour intermediate between the strong inhibitors of the type $\mathbf{B}(\mathbf{8 , 9}, \mathbf{1 1 , 1 3 )}$ and the relatively weak inhibitors of type $\mathbf{B}(\mathbf{1}-3,14-16)$. Anyhow, the best substitution for inducing strong thrombin inhibitory properties was that incorporating the 2-ethyl-4,6-diphenylpyridinium moiety in the molecules of the new derivatives. The compound containing this substitution pattern, B9, showed thrombin inhibitory potency of the same order of magnitude as the clinically used argatroban 1 and inogatran 2 , although it is less effective when compared to the very potent inhibitor NAPAP (Tables II-IV). A special mention should be made of the fact that the new compounds reported here possess a much lower affinity for trypsin than the standard inhibitors $\mathbf{1 - 3}$, which constitutes a highly desirable feature in a candidate to be developed for clinical use.

\section{QSAR Calculations}

The QSAR results are reported elsewhere' and are only summarized here. Thrombin inhibitory activity was greatly enchanced by high polarizability and large size reflected in the surface area of the molecule. Molecules containing a benzene ring bonded to the sulfonamide group were more active as both thrombin and trypsin inhibitors than the aliphatic counterparts, and there was evidence that trypsin inhibitory activity depended on the direction of the nodes in the frontier orbitals of the molecules. This dependence was different to and stronger than the corresponding result with thrombin inhibition. A major difference between the requirements for thrombin and trypsin inhibition was the effects of solvation energy, calculated as the difference in AM1 (Austin model 1) heat of formation calculated in vacuo and in aqueous solution by the conductive shielding (COSMO) model. 
It was found that trypsin inhibitory activity is favored by high solvation energy, and thrombin inhibitory activity by low. Both are favored by low lipophilicity. The most convincing results were derived not from the sulfonylaminoguanidines alone, but from the pooled data with in addition sulfonylguanidines and sulfonyl-isoureas with the same substitution patterns. ${ }^{1}$

\section{Acknowledgements}

This research was financed in part by the EU grant ERB CIPDCT 940051.

\section{References}

[1] (a) Supuran, C.T., Scozzafava, A., Briganti, F. and Clare, B.W. (1999) J. Med. Chem. (in press); (b) Clare, B.W. (1999) Theor. Chim. Acta (in press).

[2] (a) Stürzebecher, J. and Meier, J. (1995) J. Enz. Inhib., 9, 1-2; (b) Stürzebecher, J., Prasa, D., Hauptmann, J., Vieweg, H. and Wikstrom, P. (1997) J. Med. Chem., 40, 3091-3099.

[3] (a) Pavone, V., De Simone, G., Nastri, F., Galdiero, S., Staiano, N., Lombardi, A. and Pedone, C. (1998) Biol. Chem., 379, 987-1006; (b) De Simone, G., Lombardi, A., Galdiero, S., Della Morte, R., Staiano, N., Pedone, C., Bolognesi, M. and Pavone, V. (1998) Protein Sci., 7, 243-253; (c) Shafer, J.A. (1998) Curr. Opin. Chem. Biol., 2, 458-465.

[4] (a) Stubbs, M.T. and Bode, W. (1993) Thromb. Res., 69, 1-58; (b) Engh, R.A., Brandstetter, H., Sucher, G., Eichinger, A., Baumann, U., Bode, W., Huber, R., Poll, T., Rudolph, R. and von der Saal, W. (1996) Structure, 4, 1353-1362.

[5] (a) Babine, R.E. and Bender, S.L. (1997) Chem. Rev., 97, 1359-1472; (b) Salemme, F.R., Spurlino, J. and Bone, R. (1997) Structure, 5, 319-324.

[6] Eriksson, U.G., Renberg, L., Bredberg, U., Teger-Nilsson, A.C. and Regardh, C.G. (1998) Biopharm. Drug Dispos., 19, 55-64.

[7] (a) Bajusz, S., Barbas, E., Tolnay, P., Szell, E. and Bagdy, D. (1978) Int. J. Pept. Protein Res., 12, 217-221; (b) Bajusz, S., Szell, E., Bagdy, D., Barbas, E., Horvath, G., Dioszegi, M., Fittler, Z., Szabo, G., Juhasz, A., Tomori, E. and Szilagyi, G. (1990) J. Med. Chem., 33, 1729-1735.

[8] Krishnan, R., Zhang, E., Hakansson, K., Arni, R.K., Tulinsky, A., Lim-Wilby, M.S., Levy, O.E., Semple, J.E. and Brunck, T.K. (1998) Biochemistry, 37, $12094-13103$.

[9] Claeson, G., Philipp, M., Agner, E., Scully, M.F., Metternich, R., Kakkar, V.V., DeSoyza, T. and Niu, L.H. (1993) Biochem. J., 290, 309-312.

[10] (a) Stürzebecher, J., Vieweg, H., Wikstrom, P., Turk, D. and Bode, W. (1992) Biol. Chem. Hoppe-Seyler, 373, 491-496; (b) Stürzebecher, J., Prasa, D., Bretschneider, E., Bode, W., Bauer, M., Brandstetter, H., Wikstrom, P. and Vieweg, H. (1993) In DIC - Pathogenesis, Diagnosis, and Therapy of Disseminated Intravascular Fibrin Formation; (MullerBerghaus, G., Madlener, K., Blomback, M., ten Cate, J.W., Eds.), pp. 183-190. Excerpta Medica: Amsterdam, London, New York, Tokyo.

[11] (a) Adang, A.E., Lucas, H., de Man, A.P., Engh, R.A. and Grootenhuis, P.D. (1998) Bioorg. Med. Chem. Lett., 8, 3603-3608; (b) Lumma, W.C., Witherup, K.M., Tucker, T.J., Brady, S.F., Sisko, J.T., Naylor-Olsen, A.M., Lewis, S.D., Lucas, B.J. and Vacca, J.P. (1998) J. Med. Chem., 41, 1011-1013; (c) Reers, M., Koschinsky, R., Dickneite, G., Hoffmann, D., Czech, J. and Stuber, W. (1995) J. Enz. Inhib., 9, 61-72.

[12] (a) Levy, O.E., Semple, J.E., Lim, M.L., Reiner, J., Rote, W.E., Dempsey, E., Richard, B.M., Zhang, E., Tulinsky, A., Ripka, W.C. and Nutt, R.F. (1996) J. Med. Chem., 39, 4527-4530; (b) Semple, J.E., Rowley, D.C., Brunck, T.K., Ha-Uong, T., Minami, N.K., Owens, T.D., Tamura, S.Y., Goldman, E.A., Siev, D.V., Ardecky, R.J., Carpenter, S.H., Ge, Y., Richard, B.M., Nolan, T.G., Hakanson, K., Tulinsky, A., Nutt, R.F. and Ripka, W.C. (1996) J. Med. Chem., 39, 4531-4536. 
[13] Sixma, J.J. and de Groot. P.G. (1992) Thromb. Res.. 68. 507-512.

[14] Okamoto, S., Kinjo, K. Hijikata, A.. Kikumoto, R., Tamao, Y.. Ohkubo, K. and Tonomura. S. (1980) J. Med. Chem.. 23.827 . 830.

[15] (a) Stürzebecher. J., Markwardt, F., Voigt, B.. Wagner. G. and Walsmann, P. (1983) Thromb. Res.. 29,635-642; (b) Stürzebecher. J.. Markwardt, F., Voigt. B. and Wagner, G. (1984) Thromb. Res., 36, 457-467.

[16] Groutas. W.C.. Kuang, R. Venkataraman, R.. Epp. J.B.. Ruan, S. and Prakash. O. (1997) Biochemistry. 36, $4739-4750$.

[17] (a) Scozzafava. A.. Briganti. F. and Supuran. C.T. (1999) Eur. J. Med. Chem., 35, (in press): (b) Supuran. C.T., Pop, E. and Dinculescu. A. (1994) Heterocycles, 37, 667-671; (c) Supuran. C.T.. Banciu. M.D. and Balaban. A.T. (1993) Rev. Roum. Chim., 38, 199 205: (d) Supuran. C.T., Briganti. F. and Scozzafava. A. (1997) J. Enz. Inhib., 12, 175-190: (e) Briganti. F.. Pierattelli. R.. Scozzafava. A. and Supuran. C.T. (1996) Eur. J. Med. Chem. 31, $1001 \cdots 1010$.

[18] (a) Supuran. C.T. Ilies, M.A. and Scozzafava. A. (1998) Eur. J. Med. Chem., 33, 739-752; (b) Mincione. F., Menabuoni. L.. Briganti, F.. Mincione. G.. Scozzafava, A. and Supuran. C.T. (1998) J. Enz. Inhih.. 13.267-284.

[19] Winnek. P.S., Anderson, G.W., Marson, H.W.. Faith. E. and Roblin Jr., R.O. (1942) J. Am, Chem, Soc., 64, 1682-1685.

[20] Supuran, C.T.. Scozzafava. A. and Briganti. F. (1999) J. Enz. Inhib. 14, $289 \ldots 306$.

[21] Scozzafava, A. and Supuran, C.T. (1999) J. Enz. Inhih., 14. $343-363$.

[22] (a) Anderson. G.W., Zimmerman. J.E. and Cahahan, F.M. (1963) J. Am. Chem. Soc., 85 , 3039: (b) Sheehan. J.C. and Ledis. S.L. (1973) J. Am. Chem. Soc., 95, 875 879: (c) Zlatoidsky, P. and Maliar. T. (1996) Eur. J. Med. Chem.. 31, 895--900.

[23] Lottenberg. R.. Christensen. U., Jackson. C.M. and Coleman. P.L. (1981) Meth. Enzymol, 80. $341 \cdots 361$.

[24] Hemker. H.C.. Willems, G.M. and Beguin, S. (1986) Thromh. Haemostas., 56, $9 \ldots 17$.

[25] Gaussian 94 (Revision A.1), M.J. Frisch, G.W. Trucks, H.B. Schlegel, P.M.W. Gill, B.G. Johnson. M.A. Robb. J.R. Cheeseman. T.A. Keith. G.A. Petersson, J.A. Montgomery. K. Raghavachari. M.A. Al-Laham. V.G. Zakrzewski, J.V. Ortiz, J.B. Foresman, C.Y. Peng. P.Y. Ayala, M.W. Wong. J.L. Andres, E.S. Replogle, R. Gomperts, R.L. Martin. D.J. Fox, J.S. Binkley. D.J. Defrees. J. Baker. J.P. Stewart. M. Head-Gordon, C. Gonzalez and J.A. Pople (1995). Gaussian. Inc.: Pittsburgh PA.

[26] MOPAC 93.00, Stewart J.J.P. (1993) Fujitsu Ltd.; Tokyo, Japan, also Stewart J.J.P. (1995) Q.C.P.E. Bull.. 15. 13 14 (Copyright Fujitsu 1993, all rights reserved).

[27] Hyperchem 1. 5.1. Available from Hypercube. Inc. $1115 \mathrm{NW}$ 4th Street, Gainesville, Florida 32601-4256. USA.

[28] Dewar, M.J.S., Zoebisch. E.G.. Healy. E.F. and Stewart. J.J.P. (1985) J. Am. Chem. Soc., 107. 3902-3909.

[29] Klamt, A. and Shuurmann. G. (1993) J. Chem. Soc. Perkin Trans., 799-805.

[30] Lee. C.. Yang. W. and Parr. R.G. (1988) Phys. Rev. B. 37. 785-789.

[31] Parr. R.G. and Yang. W. (1989) Density-Functional Theory of Atoms and Molecules. Oxford Univ. Press: Oxford.

[32] Foresman, J.B.. Keith. T.A.. Wiberg. K.B.. Snoonian. J. and Frisch. M.J. (1996) J. Phy's. Chem. 100. 16098 16104.

[33] Pallas for Windows. version $3.1 \mathrm{pK}_{\text {a }}$ Prediction Module. pKalc 3.1: Available from CompuDrug Chemistry Ltd. H-1395 Budapest, 62 P.O.B. 405, Hungary.

[34] Banner. D.W. and Hadvary. P. (1991) J. Biol. Chem.. 266. $20085-20093$.

[35] Balasubramanian, N., Laurent. D.R.S.. Federici, M.E.. Meanwell, N.A., Wright, J.J., Schumacher, W.A. and Seiler, S.M. (1993) J. Med. Chem, 36. 300-303.

[36] (a) Bode. W.. Turk. D. and Stürzebecher, J. (1990) Eur. J. Biochem., 193, 175--182; (b) Morenweiser. R., Auerswald. E.A., van de Locht. A.. Fritz, H.. Stürzebecher, J. and Stubbs. M.T. (1997) J. Biol. Chem.. 272. $19938 \cdots 19942$.

[37] Supuran, C.T.. Manole. G.. Dinculescu. A., Schiketanz. A.. Gheorghiu, M.D., Puscas, I. and Balaban. A.T. (1992) J. Pham. Sci., 81, 716 719.

[38] Supuran, C.T.. Scozzafava. A.. Ilies. M.A.. Iorga. B.. Cristca, T.. Chiraleu, F. and Banciu. M.D. (1998) Ew. J. Med. Chem. 33. $577 \quad 594$. 